Review Article

\title{
A Review of Peridynamics (PD) Theory of Diffusion Based Problems
}

\author{
Migbar Assefa Zeleke $\mathbb{i D}^{1,2}$ and Mesfin Belayneh Ageze $\mathbb{i D}^{3}$ \\ ${ }^{1}$ Department of Mechanical Engineering, Institute of Technology, Hawassa University, Hawassa, Ethiopia \\ ${ }^{2}$ Department of Mechanical Engineering, University of Botswana, Gaborone 0061, Botswana \\ ${ }^{3}$ Center of Renewable Energy, Addis Ababa Institute of Technology, Addis Ababa University, Addis Ababa, Ethiopia
}

Correspondence should be addressed to Migbar Assefa Zeleke; migbarassefa@gmail.com

Received 27 July 2021; Accepted 1 October 2021; Published 19 October 2021

Academic Editor: Roberto Brighenti

Copyright ( 92021 Migbar Assefa Zeleke and Mesfin Belayneh Ageze. This is an open access article distributed under the Creative Commons Attribution License, which permits unrestricted use, distribution, and reproduction in any medium, provided the original work is properly cited.

\begin{abstract}
The study of heat conduction phenomena using peridynamic (PD) theory has a paramount significance on the development of computational heat transfer. This is because PD theory has got an interesting feature to deal with the inherent nonlocal nature of heat transfer processes. Since the revolutionary work on PD theory by Silling (2000), extensive investigations have been devoted to PD theory. This paper provides a survey on the recent developments of PD theory mainly focusing on diffusion based peridynamic (PD) formulation. Both the bond-based and state-based PD formulations are revisited, and numerical examples of two-dimensional problems are presented.
\end{abstract}

\section{Introduction}

Modeling and simulation of diffusion constitute an essential process we usually come across in our everyday life and in a number of industrial processes. The basic and fundamental knowledge of diffusion process is vital in the efficient utilization of energy in different cooling and heating processes. The governing mathematical equations of many physical phenomena in engineering and science are described by local diffusion equations. In solving these equations, researchers employed several numerical techniques in the past several years including finite element method (FEM), boundary element method (BEM) [1], finite difference method (FDM), and meshless methods. At the continuum level, diffusion processes are typically defined by local models via the famous Fourier's law of heat conduction and Fick's law of mass transport. The main drawback of the local models is that they ignore the nonlocal effect. In fact, the effect of nonlocality cannot be ignored due to the fact that nonlocality plays a significant role in diffusion processes like heat conduction, moisture flow, electrical conduction, pitting corrosion, and hydraulic fracturing. Hence, it is quite critical to develop a nonlocal model that accommodates both the nonlocality effect and emerging discontinuities at the same time.

In this regard, peridynamics (PD) is a promising and suitable approach for dealing with the aforementioned challenges encountered by the local models. PD theory was proposed by Silling [2]. It was mainly intended to deal with solid mechanics problems, but later it was effectively extended and realized to solve diffusion related problems. An interesting breakthrough that proves that PD is beyond solid mechanics problems was presented by Gerstle et al. [3]. In this article, the authors showed the applicability of PD in solving multiphysics problems like electromigration. The beauty of PD theory is that it takes into account both nonlocal interactions and constitutive laws simultaneously. Later, Bobaru and Duangpanya [4] developed a 1D heat conduction PD formulation, and then they extended it to $2 \mathrm{D}$ heat conduction problems with discontinuities [5]. On the other hand, [6] compared and analyzed various discretization schemes of nonlocal diffusion (ND) and linear PD equations by demonstrating illustrative approaches developed for solving nonlocal models with both 
nonintegrable and integrable kernels. In this article the authors paid attention to conforming finite element and quadrature-based finite difference techniques in the discretization of PD and ND models. Following the pioneering works of Bobaru and Duangpanya [4, 5], Agwai [7] and Oterkus et al. [8] pushed further the bond-based PD formulation developed by $[4,5]$ to the state-based PD formulation. The formulation developed by $[7,8]$ is so general and can be reduced to the bond-based formulations of $[4,5]$. The only difference between the bond-based formulations in $[4,5,7,8]$ is that they use different response functions (kernel functions) as briefly described by Chen and Bobaru [9].

PD formulation for saturated steady-state pressure driven porous flow has been demonstrated by Katiyar et al. [10]. Later Jabakhanji and Mohtar [11] extended the work of [10] to transient moisture flow in unsaturated, anisotropic, and heterogeneous soils in PD framework. A PD diffusion model by employing Green's function has been developed by [12]. In this study, the steady-state and transient PD Green's functions were derived and implemented to $2 \mathrm{D}$ infinite plate which is heated by a Gauss source. PD formulation of heat conduction in functionally graded materials (FGMs) has been developed by Liao et al. [13]. The authors in this article employed a state-based peridynamic (PD) approach to simulate heat conduction phenomena with insulated cracks in FGMs. In the same year [14], addressed the issue of transient heat transfer by combining the classical and PD formulations simultaneously. In this study, the integrodifferential equations have been solved by employing the spectral technique based Galerkin scheme. Another contribution in relation to PD diffusion problems by employing a general-purpose finite element analysis software ANSYS was from [15]. The technique implemented in this article was interesting in that it reduced the computational time significantly due to the fact that the authors used implicit time integration scheme.

The transient advection diffusion PD model has been presented by Zhao et al. [16] by extending the bond-based PD approach developed by Bobaru and Duangpanya [5]. Xue et al. [17] on the other hand addressed thermal contact problems by utilizing the state-based version of peridynamic formulation. In this article, the authors employed the domain decomposition technique to solve heat transfer problems by considering the thermal flux and temperature as the primary variables. Recently, [18] applied PD differential operator to study a refined bond-based PD heat conduction model by reviewing the present state-based and bond-based PD heat conduction models. By extending the work of [13], Tan et al. [19] performed their research on heat conduction in FGMs with discontinuities using PD by taking into consideration the effect of surface correction near the crack and domain boundaries. Later, [20] investigated the effects of interfacial transition zone properties and diffusivity on chloride diffusion concrete of arbitrarily distributed aggregates using multiscale PD model. To improve the computational precision and diminish the cost of computation, the authors employed multiscale discretization scheme.
PD diffusion model with a capability of handling unbounded domains using accurate absorbing boundary conditions ( $\mathrm{ABCs}$ ) has been developed by [21]. In this study, the authors employed mesh-free discretization scheme in case of PD diffusion models whereas FEM has been employed in the case of local diffusion models. Wang et al. [22] very recently developed a dual horizon PD formulation to study thermal diffusion problem. The Lagrangian formulation has been implemented in this study to develop the governing equations. One of the benefits of this formulation is that it permits the implementation of flexible discretization in the domain of interest which in turn contributes a lot to the reduction of computational time. Yan et al. [23] further implemented the BB-PD formulation to model a coupled chemical transport and water flow in unsaturated discontinuous and heterogeneous media. In this study, processes like dispersion, diffusion, and advection have been considered in partially saturated porous media.

The main objective of this article is to revisit recent developments in PD applications that are pertinent to diffusion-type problems. Therefore, it is structured as follows. The first section introduces and reviews PD theory in general. The second section is devoted to PD formulation of diffusion-type problems; it is segmented into PD heat conduction, PD electrical conduction, and PD moisture flow. Finally, several illustrative examples of different cases are presented, and the performance of PD is investigated.

\section{Review of Peridynamic Theory}

Diffusion related PD formulation cannot be understood without understanding its uniqueness as compared with classical continuum theory. In classical continuum mechanics, material particles interact with the nearby immediate material particles as shown in Figure 1(a). In contrast to the classical continuum theory, PD material points are allowed to interact with material points within its family $\left(H_{i}\right)$ at a finite distance called horizon as shown in Figure 1(b).

PD theory is a nonlocal integrodifferential mesh-free method without spatial derivatives $[2,24]$. It is just the nonlocal version of classical continuum theory; its formulation mainly depends on an integrodifferential equation unlike the classical counterpart, which is based on spatial derivatives $[24,25]$. The term peridynamics was originally coined by Silling [2] at Sandia National Laboratories in the late nineties. This paper reformulated the continuum based equation of motion (EOM) to integrodifferential equation to deal with spontaneous emergence and propagation of discontinuity in solids. For the PD formulation of elasticity, consider a body having a region $\Omega$ as shown in Figure 2 . With reference to Figure 1, the EOM for particle $i$ at time $t$ as proposed in [2] is given as (1). The original formulation in this paper [2] was the bond-based PD theory, where internal forces in a body are modeled as a network of pairwise interactions. The material points interact in a pairwise manner and are restricted to a specified neighborhood through a bond. The force of interaction between a pair of material points is dependent on the deformation of the two points only. 


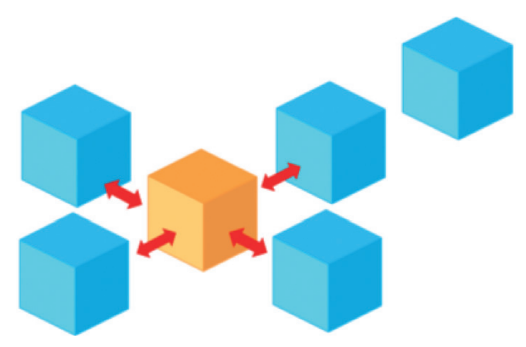

(a)
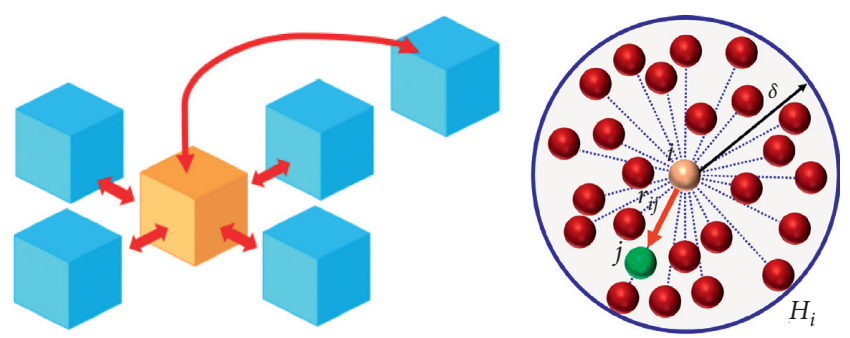

(b)

Figure 1: Representations of local and nonlocal interaction: (a) local theory; (b) nonlocal theory.

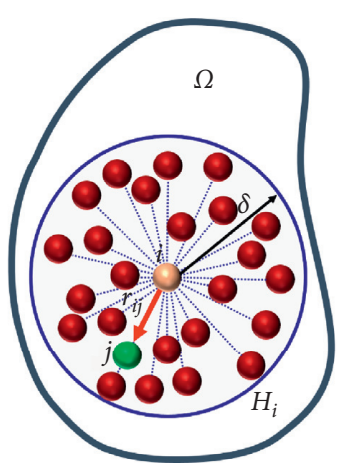

(a)

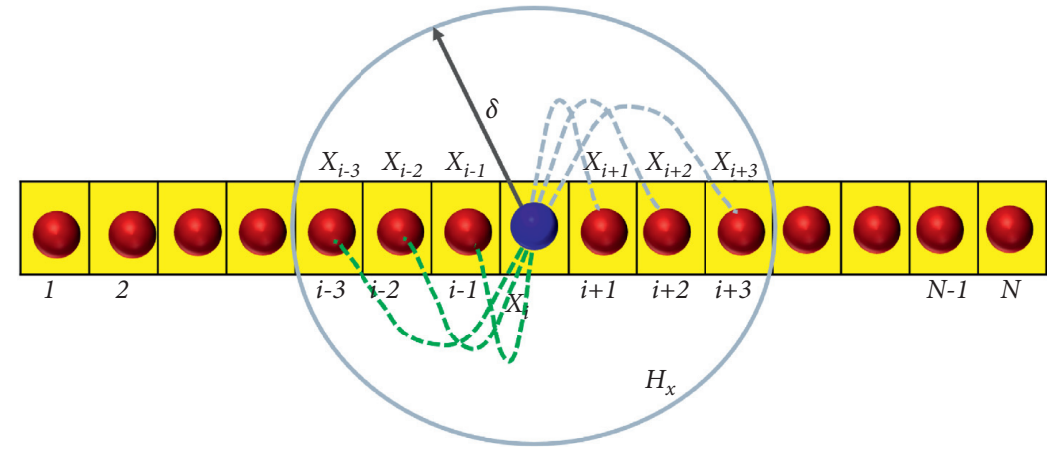

(b)

FIgURE 2: Peridynamics concept representation: (a) peridynamic medium representation; (b) 1D peridynamic medium representation.

$$
\rho_{i} \ddot{\mathbf{u}}(i, t)=\int_{H_{x}} f\left(\mathbf{u}_{j}-\mathbf{u}_{i}, \mathbf{r}_{i j}\right) \mathrm{d} V_{j}+\mathbf{b}[i, t],
$$

where $\rho$ is mass density, $\mathbf{u}$ is displacement vector field, $\mathbf{f}$ is pairwise force function (the force vector per unit square of volume that the material point $j$ exerts on material point $i$ ), b is a body force density, $H_{i}$ is a neighborhood of particle $\mathrm{i}$ with radius $\delta$ as shown in Figure $2, \mathbf{r}_{i j} \leq \delta$ is the position vector of the nearest particle pointing to $i$ from $j$, and $\mathrm{d} V_{i}$ is the differential volume of $j$ inside the horizon of $i$.

Since its introduction, PD theory has been used to solve a variety of problems. Accordingly, literature concerning peridynamic theory is quite exhaustive and abundant. In the past two decades, several peridynamic studies pertaining to elastic deformation and fracture solids [2, 26-28], brittle fracture [29-33], fatigue failure [34-49], and PD application of damage in composites [33, 43, 50-65] have been reported. Studies related to crack initiation and propagation using peridynamics can be found in [30, 66-69]. As far as studies related to plasticity, viscoelasticity, and viscoplasticity are concerned, the reader is advised to refer to [70-81]. Figure 3 shows the general trend in the number of articles published in the referenced journals. Figure 4 on the other hand shows the citation history of the first pioneering article [2] in the area of PD theory since its first publication. It is evident from these two figures (Figures 3 and 4) that year after year research outputs pertinent to PD theory increase incredibly.

Therefore, the aim of this section is to provide an overview on the computational aspects of PD theory with emphasis on the modeling of diffusion problems. In this regard, the citation trends of the most influential and pioneering articles in the areas of PD based diffusion models are presented as shown in Figure 5 [4, 8]. Figure 5 compared citation trends of the bond-based PD thermal diffusion developed by $[4,5]$ and state-based PD thermal diffusion formulation of [8]. Similar observations may be realized from Figure 5 too.

Therefore, this section is outlined as follows: the first part provides a review of the state-based PD formulation of heat conduction, followed by electrical conduction and moisture flow. The discussion in this paper mainly revisits the statebased peridynamic theory, and then later we will reduce the SB equations to bond-based (BB) equations as the particular case of state-based peridynamic theory. Although the nonlocal model we propose in this review offers a better predictive capability of high strain gradient and fracture mechanics, the scope of this review is limited to diffusion based problems only.

2.1. State-Based PD Formulation for Heat Conduction. In general, heat transfer is directly related to temperature whether it is radiation, convection, or conduction. Heat conduction is the process by which temperature gradient exists within a body; hence, its primary objective is to determine temperature distribution and exchange of energy within a body. The development of numerical solutions for heat conduction problems is growing as an effective tool in thermal engineering. The applicability of continuum based heat transfer equations to bodies with discontinuities is mathematically awkward due to the fact that these equations 


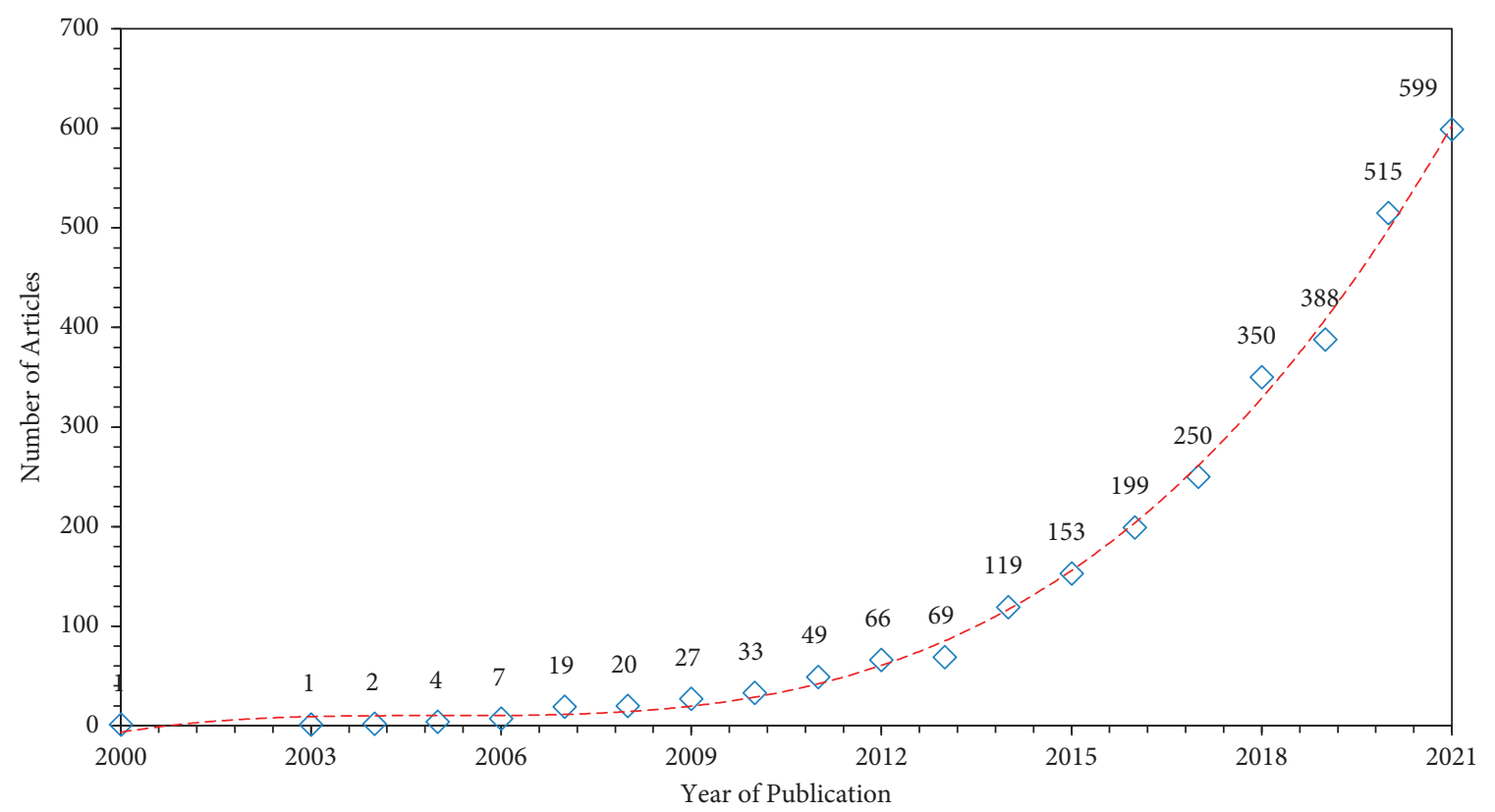

Figure 3: Number of publications per year from Scopus database.

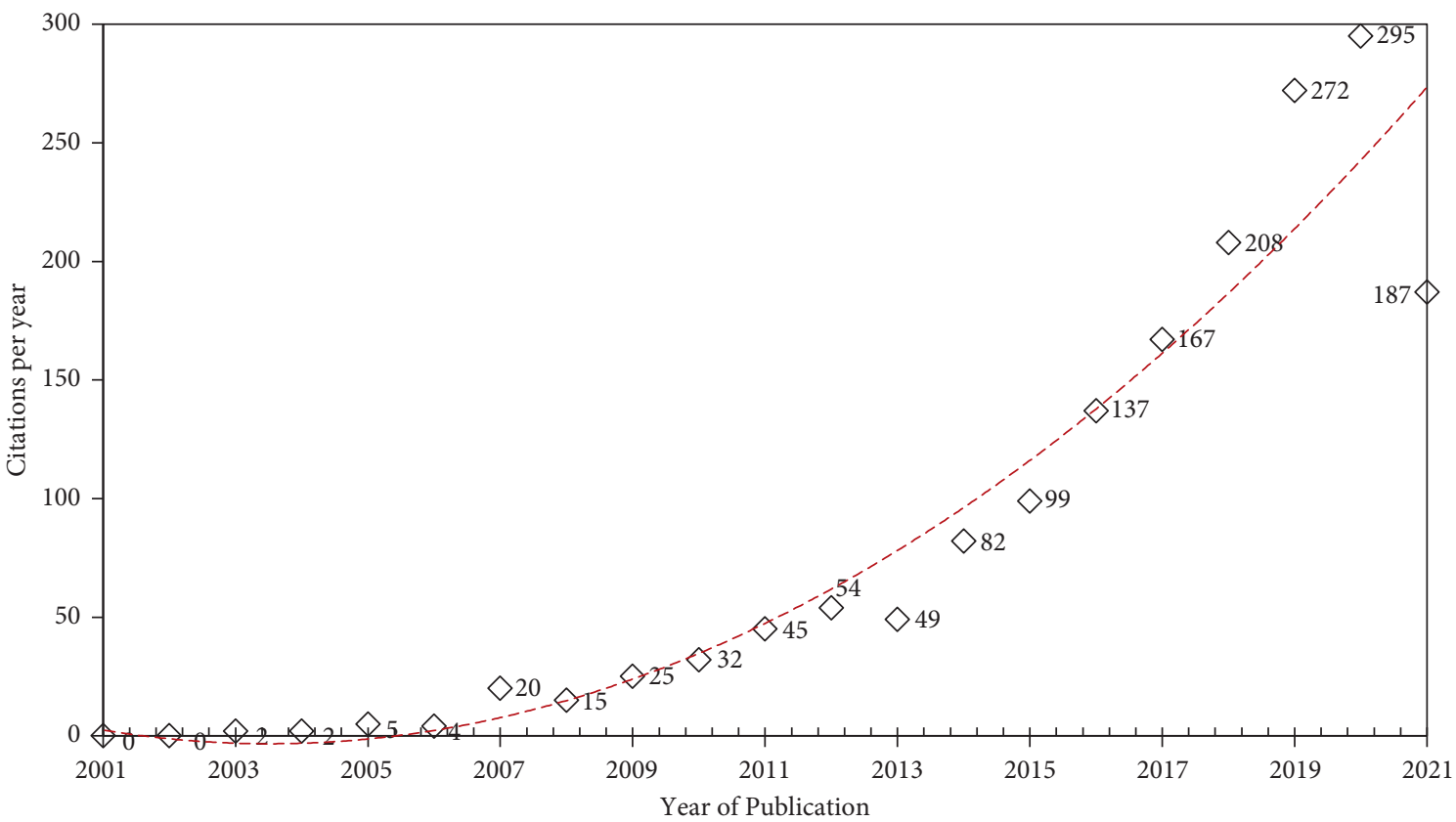

Figure 4: Publication history of [2] from Scopus database.

contain temperature gradients. To deal with the aforementioned challenges and limitations of the continuum based heat transfer equations, PD theory gives ideal solution. This section covers PD formulation of heat conduction equations. The PD heat conduction equations used in this article are simple and interesting in that thermal conductivity coefficient of a particle is defined within its neighborhood at a certain finite distance called horizon [9], unlike continuum based Fourier theory where a particle interacts only with its immediate vicinity. Hence, PD is a valuable tool that takes into account both the nonlocality effect and discontinuities concurrently across the temperature and other potential fields like electric potential, hydraulic potential, and chemical potential.

The present section addresses studies that are pertinent to heat transfer. Gerstle et al. [3] were the first to propose the analytical and computational simulation of electromigration that accounts for heat transfer in a one-dimensional problem. Later, Bobaru and Duangpanya [4] introduced the bond-based PD formulation for thermal problems with 


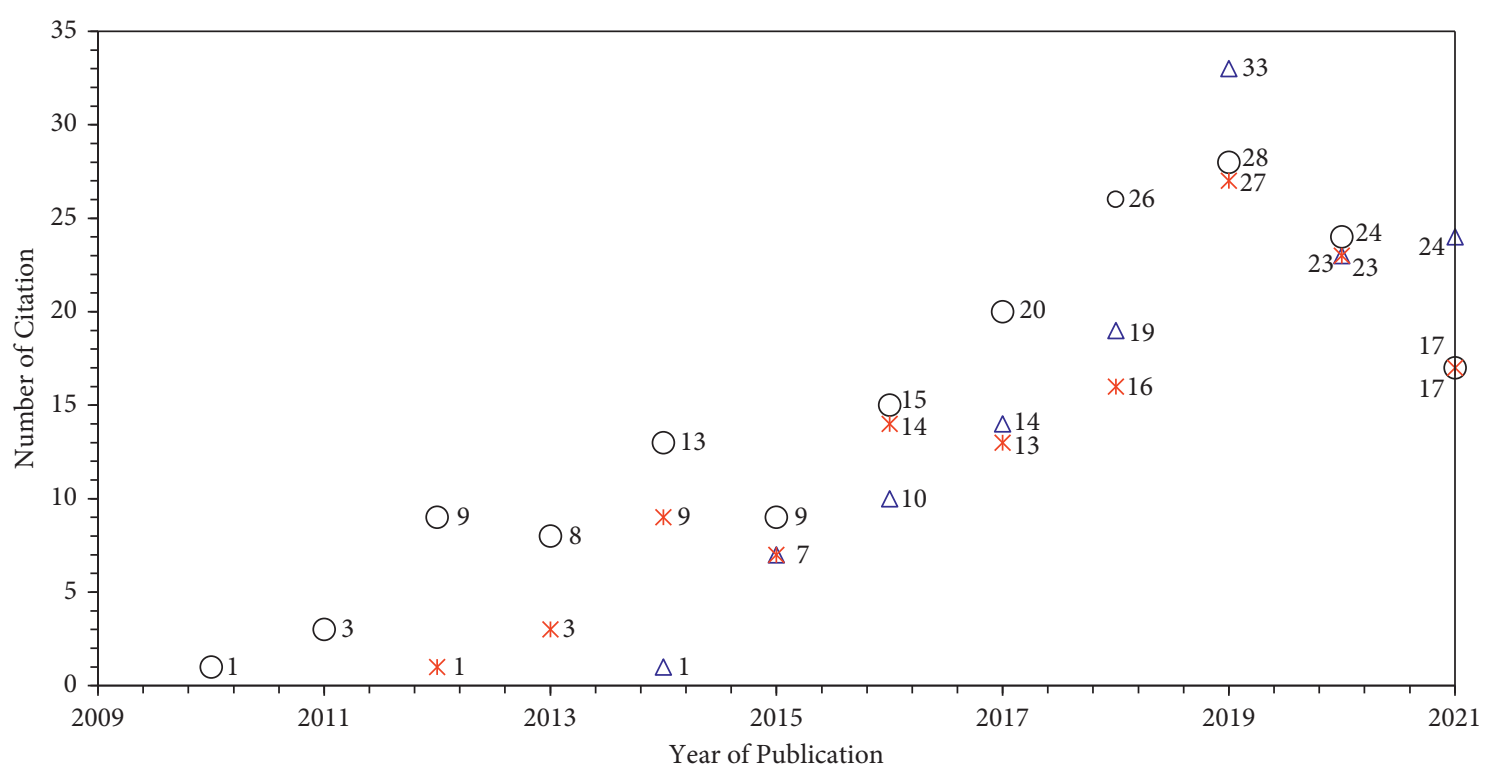

\footnotetext{
$\triangle$ Oterkus S., Madenci E., Agwai A._2014

○ Bobaru F., Duangpanya M._2010

* Bobaru F., Duangpanya M._2012
}

Figure 5: Publication history comparison between authors of $[4,5,8]$ from Scopus database.

evolving discontinuities. The authors used a constructive approach to obtain the PD equations for heat transfer. Following their previous contribution [4], Bobaru and Duangpanya [5] proposed a multidimensional PD formulation to solve two-dimensional heat conduction problems with discontinuities. Recently, the generalized state-based PD heat transfer problem using Lagrangian approach was demonstrated by Oterkus et al. [8]. In this work, the authors determined the PD material parameter, the micro conductivity, by simplifying the state-based PD heat transfer equation to its bond-based PD heat transfer equation. The authors also confirmed that the governing equation represented the conservation of thermal energy. Later, Chen and Bobaru [9] analyzed the behavior of PD solutions for transient heat diffusion model and studied the convergence properties of the one point Gauss quadrature scheme.

In PD heat conduction, the interaction among material points is due to the exchange of heat. Hence, we present the governing PD equations based on the one proposed by Oterkus et al. [8] and Agwai [7] for the sake of completeness by considering temperature as primary variable.

Based on the generalized state-based PD formulation, the temperature state $\underline{\tau}$ that contains the temperature difference associated with each interaction of a particular material point is given by

$$
\underline{\tau}_{i}\left\langle\mathbf{r}_{i j}\right\rangle=T_{j}-T_{i},
$$

where $T$ is the temperature. Note that states are represented by variables with underscores, and the angular brackets indicate the bond being operated on.
According to $[7,8]$, the SBPD heat flow state is written as

$$
\underline{h}=\underline{h}(\underline{\tau}) .
$$

Therefore, the transient form of heat conduction in the framework of SBPD is expressed as $[7,8]$

$$
\left(\rho C_{v}\right)_{i} \dot{T}_{i}=\int_{H_{i}}\left(\underline{h}_{i}\left\langle\mathbf{r}_{i j}\right\rangle-\underline{h}_{j}\left\langle\mathbf{r}_{j i}\right\rangle\right) \mathrm{d} V_{j}+s_{i},
$$

where $\underline{h}=\mathbf{q}^{T} \mathbb{K} \mathbf{r}_{i j} w_{i j}$

Therefore, (4) may be modified as follows:

$$
\left(\rho C_{v}\right)_{i} \dot{T}_{i}=\int_{H_{i}}\left(\mathbf{q}_{j}^{T} \mathbb{K}_{j}+\mathbf{q}_{i}^{T} \mathbb{K}_{i}\right) \mathbf{r}_{i j} w_{i j} \mathrm{~d} V_{j}+S_{i}
$$

where $s_{i}$ is the heat sink or source, $\mathbf{q}$ is the classical heat flux, and $C_{v}$ is the specific heat capacity.

For PD heat conduction phenomena, the discrete form of (5) may be expressed in the form of finite sum as follows:

$$
\begin{aligned}
\therefore\left(\rho C_{v}\right)_{i} \dot{T}_{i} & =\underbrace{\sum_{j \in H_{i}}\left(\mathbf{q}_{j}^{T} \mathbb{K}_{j}+\mathbf{q}_{i}^{T} \mathbb{K}_{i}\right) \mathbf{r}_{i j} w_{i j} V_{j}}_{-\nabla \cdot q_{i}}+s_{i}, \\
\nabla \cdot \mathbf{q}_{i} & \longrightarrow \sum_{j \in H_{i}}\left(\mathbf{q}_{j}^{T} \mathbb{K}_{j} \mathbf{r}_{j i}-\mathbf{q}_{i}^{T} \mathbb{K}_{i} \mathbf{r}_{i j}\right) w_{i j} V_{j},
\end{aligned}
$$

where $\mathbb{K}_{i}$ is shape tensor related to thermal field and it is given as follows: 


$$
\begin{aligned}
\mathbb{K}_{i}= & {\left[\sum_{j \in H_{i}} \mathbf{r}_{i j} \otimes \mathbf{r}_{i j} w_{i j} V_{j}\right]^{-1}, } \\
H= & {\left[\sum_{j \in H_{i}}\left(T_{j}-T_{i}\right) \mathbf{r}_{i j} w_{i j} V_{j}\right], } \\
\therefore \nabla T_{i}= & \mathbb{K}_{i} \cdot H \longrightarrow\left[\sum_{j \in H_{i}} \mathbf{r}_{i j} \otimes \mathbf{r}_{i j} w_{i j} V_{j}\right]^{-1} \\
& \cdot\left[\sum_{j \in H_{i}}\left(T_{j}-T_{i}\right) \mathbf{r}_{i j} w_{i j} V_{j}\right],
\end{aligned}
$$

where $\quad w_{i j}=\left\{\begin{array}{l}1, \mathbf{r}_{i j} \leq \delta \\ 0, \mathbf{r}_{i j}>\delta\end{array}\right.$

and

$\nabla T_{i}$ is the gradient of temperature.

2.1.1. Correlations between the Classical Heat Flux and PD Heat Flow State. The heat flow scalar state $\underline{h}$ contains the heat flow densities associated with all the interactions $[7,8]$. Therefore, heat flow density $\underline{h}$ has units of heat flow rate per volume square.

$$
\int_{H_{i}}\left(\underline{h}_{i}\left\langle\mathbf{r}_{i j}\right\rangle-\underline{h}_{j}\left\langle\mathbf{r}_{j i}\right\rangle\right) \mathrm{d} V_{j}
$$

Equation (9) resembles the divergence of heat flux $\nabla \cdot \mathbf{q}$, which has units of heat flow rate per volume and is given in the above equation.

Therefore, the PD heat flow state can be correlated to the heat flux q. Then, the expression that relates the heat flux to the heat flow state has been borrowed from $[7,8]$.

\subsubsection{Bond-Based Peridynamic (PD) Heat Conduction} Formulation. In a bond-based peridynamic model, material point $i$ can interact with all neighboring material points $j$ in its horizon in a pairwise manner. The change in temperature at the two end points of a bond is assumed to cause the heat to flow along the central axis of the bond only. When material points interact in a pairwise manner and are restricted to a specified neighborhood through a bond, eq (6) may be reduced as follows:

$$
\begin{aligned}
& \left(\rho C_{v}\right)_{i} \dot{T}_{i}=\int_{H_{i}} f_{h} \mathrm{~d} V_{j}+S_{i}, \\
& \left(\rho C_{v}\right)_{i} \dot{T}_{i}=\int_{H_{i}}\left(k \frac{T_{j}-T_{i}}{\mathbf{r}_{i j}}\right) \mathrm{d} V_{j}, \\
& f_{h}\left(\mathbf{r}_{i j}, t\right)=k \frac{T_{j}-T_{i}}{\mathbf{r}_{i j}},
\end{aligned}
$$

where $k=\widehat{\kappa} / V_{H_{i}}$ is micro conductivity of the connected thermal bonds that joins point $i$ and $j$ as shown in Figure 2, $V_{H_{i}}$ is the horizon volume of material point centered at $i$, and $\widehat{\kappa}$ is the PD conductivity of thermal bonds between material points $i$ and $j$.
2.1.3. Linking Peridynamic Properties with Those of the Classical Counterparts. In order to create a relationship between the PD properties and the standard material properties, we borrow directly expressions from [8]. Thus, for one-, two-, and three-dimensional analysis, the PD thermal micro conductivities are expressed correspondingly as

$$
\begin{cases}k=\frac{2 \kappa}{A \delta^{2}}, & \text { for }(1-D), \\ k=\frac{6 \kappa}{\pi h \delta^{3}}, & \text { for }(2-D), \\ k=\frac{6 \kappa}{\pi h \delta^{4}}, & \text { for }(3-D),\end{cases}
$$

where $\delta, A, \kappa$, and $h$ are horizon, cross-sectional area, thermal conductivity, and thickness, respectively.

\subsection{State-Based PD Formulation for Electrical Conduction.} The present section deals with the PD formulation of electrical conduction. Articles that are pertinent to electrical conduction phenomena alone are quite limited, but there are few about the coupled form. The application of PD to the failure of dielectric solids can be found in [82]. On the other hand, Prakash and Seidel [83] explained the effectiveness of PD model in examining the piezoresistive composite materials. The same authors further developed an electromechanical PD model to predict the deformation and damage of explosive materials [84, 85]. Zeleke et al. [86-88] on the other hand developed a PD formulation for thermoelectric phenomena. A recent contribution of Diana and Carvell [89] employed micropolar PD (MPPD) model to solve electromechanical problems. Very recently, Zeleke et al. [88] employed PD theory to study discontinuities in electric and thermal fields.

Therefore, in this section, we first describe PD electrical conduction using the generalized state-based approach. The derivation of the generalized PD electrical conduction equation is established, and the peridynamic variables are elucidated. Afterward, simplifications are made, so that the bond-based PD formulation for electrical conduction could be developed.

For electrical conduction phenomena, material points exchange electrical current with points inside its neighborhood defined by the horizon. In this section, we derived the state-based PD electrical conduction equation by employing a variational technique like Katiyar et al. [10] for pressure driven porous flow. Based on state-based PD formulation, the potential state $\varphi$ that comprises the electrical potential difference linked with each interaction of a particular material point is given by

$$
\underline{\varphi}_{i}\left\langle\mathbf{r}_{i j}\right\rangle=\Phi_{j}-\Phi_{i}
$$

where $\Phi$ is the electric potential.

Therefore, the governing equation for electrical conduction in the framework of SBPD is obtained as 


$$
\dot{\varrho}_{i}=\int_{H_{i}}\left(\underline{Q}_{i}\left\langle\mathbf{r}_{i j}\right\rangle-\underline{Q}_{j}\left\langle\mathbf{r}_{j i}\right\rangle\right) \mathrm{d} V_{j}+J_{i},
$$

where $Q_{i}$ is the electrical current flow state, $\dot{\varrho}_{i}$ is the time rate of charge density, and $J_{i}$ is the charge source. By extending the SBPD heat flow state, we can write the state-based current flow state as

$$
\underline{Q}=\underline{Q}(\underline{\varphi})
$$

where $\underline{\phi}_{i}$ is $\mathrm{PD}$ electrical potential scalar state and $\underline{Q}$ is current flow state; $\underline{Q}=\mathbf{j}^{T} \mathbb{K} \mathbf{r}_{i j} w_{i j}$.

$$
\dot{\varrho}_{i}=\int_{H_{i}}\left(\mathbf{j}_{j}^{T} \mathbb{K}_{j}+\mathbf{j}_{i}^{T} \mathbb{K}_{i}\right) \mathbf{r}_{i j} w_{i j} \mathrm{~d} V_{j}+J_{i},
$$

where $\mathbf{j}$ is classical current flux and $J_{i}$ is charge source.

Equation (18) in its discrete form may be written as

$$
\therefore \dot{\varrho}_{i}=\underbrace{\sum_{j \in H_{i}}\left(\mathbf{j}_{j}^{T} \mathbb{K}_{j}+\mathbf{j}_{i}^{T} \mathbb{K}_{i}\right) \mathbf{r}_{i j} w_{i j} V_{j}}_{-\nabla \cdot j_{i}}+J_{i},
$$

where $\left(\mathbb{K}_{E L}\right)_{i}$ is shape tensor related to electrical field and it is given as follows:

$$
\begin{aligned}
\left(\mathbb{K}_{E L}\right)_{i}= & {\left[\sum_{j \in H_{i}} \mathbf{r}_{i j} \otimes \mathbf{r}_{i j} w_{i j} V_{j}\right]^{-1}, } \\
H_{E L}= & \sum_{j \in H_{i}}\left(\Phi_{j}-\Phi_{i}\right) \mathbf{r}_{i j} w_{i j} V_{j}, \\
\therefore \nabla \Phi_{i}= & \left(\mathbb{K}_{E L}\right)_{i} \cdot H_{E L} \longrightarrow\left[\sum_{j \in H_{i}} \mathbf{r}_{i j} \otimes r_{i j} w_{i j} V_{j}\right]^{-1} \\
& \cdot\left[\sum_{j \in H_{i}}\left(\Phi_{j}-\Phi_{i}\right) \mathbf{r}_{i j} w_{i j} V_{j}\right] .
\end{aligned}
$$

\subsubsection{Relationship between Electrical Current Density and PD} Current Flow State. The current flow scalar state $Q$ comprises the current flow densities linked with all the participating material points having units of current flow per unit volume square and given as

$$
\int_{H_{i}}\left(\underline{Q}_{i}\left\langle\mathbf{r}_{i j}\right\rangle-\underline{Q}_{j}\left\langle\mathbf{r}_{j i}\right\rangle\right) \mathrm{d} V_{j}
$$

Equation (22) resembles the divergence of electric flux $\nabla \cdot \mathbf{j}$, which has units of charge flow rate per volume and is given as

$$
\nabla \cdot \mathbf{j}_{i} \longrightarrow \sum_{j \in H_{i}}\left(\mathbf{j}_{j}^{T} \mathbb{K}_{j} \mathbf{r}_{j i}-\mathbf{j}_{i}^{T} \mathbb{K}_{i} \mathbf{r}_{i j}\right) w_{i j} V_{j}
$$

By extending the PD heat flow state formulation to the current flow state, we may have the expression that relates the current flux to the current flow state as follows:

$$
\begin{aligned}
& \underline{Q}=\mathbf{j}^{T} \mathbb{K} \mathbf{r}_{i j} w_{i j}, \\
& \underline{Q}=\left(\mathbf{j}_{j}^{T} \mathbb{K}_{j}+\mathbf{j}_{i}^{T} \mathbb{K}_{i}\right) \mathbf{r}_{i j} w_{i j}
\end{aligned}
$$

2.2.2. Bond-Based Peridynamic (PD) Electrical Conduction Formulation. In a bond-based peridynamic model, material point $i$ can interact with all neighboring material points $j$ in its horizon in a pairwise manner. The change in electric potential at the two points of a bond is assumed to cause the electric current to flow along the axis of the bond only, which results in pairwise interaction of material points. Therefore, (19) may be reduced as follows:

$$
\begin{aligned}
\dot{\varrho}_{i} & =\int_{H_{i}} f_{I} \mathrm{~d} V_{j}+J_{i}, \\
\dot{\varrho}_{i} & =\int_{H_{i}}\left(k_{E} \frac{\Phi_{j}-\Phi_{i}}{\mathbf{r}_{i j}}\right) \mathrm{d} V_{j}, \\
\underline{\varphi}_{i} \mathbf{r}_{i j} & =\Phi_{j}-\Phi_{i}, \\
f_{I}\left(\mathbf{r}_{i j}, t\right) & =k_{E} \frac{\Phi_{j}-\Phi_{i}}{\mathbf{r}_{i j}},
\end{aligned}
$$

where $k_{E}=\widehat{\kappa}_{E} / V_{H_{i}}$ is micro conductivity of the associated electrical bonds that connect points $i$ and $j$ as shown in Figure 2, $V_{H_{i}}$ is the horizon volume of material point centered at $i$, and $\widehat{\kappa}_{E}$ is the PD conductivity of electrical bonds between material points $i$ and $j$.

2.2.3. Linking Peridynamic Properties with Those of the Classical Counterparts. In order to create a connection between the PD properties and the classical material properties, we directly borrow expressions from [8] and extend them to electric field. Thus, for one-, two-, and threedimensional analysis, the PD electrical micro conductivities are expressed as follows:

$$
\begin{cases}k_{E}=\frac{2 \kappa_{E}}{A \delta^{2}} ; & \text { for }(1-D), \\ k_{E}=\frac{6 \kappa_{E}}{\pi h \delta^{3}} ; & \text { for }(2-D), \\ k_{E}=\frac{6 \kappa_{E}}{\pi h \delta^{4}} ; & \text { for }(3-D),\end{cases}
$$

where $\delta, A, \kappa_{E}$, and $h$ are horizon, cross-sectional area, electrical conductivity, and thickness, respectively.

2.3. State-Based PD Formulation for Chemical and Water Transport. In this section, the PD models of chemical transport and water flow in their uncoupled state were revisited. In the realm of PD theory, a number of scholars addressed the issue of diffusion processes in both saturated and unsaturated porous media. Katiyar et al. [10] established 
a PD model to study the steady-state water flow in saturated porous media by taking into account the effect of heterogeneities and discontinuities. Later, Jabakhanji and Mohtar [11] addressed the transient nature of moisture flow in unsaturated porous media in PD framework. Very recently, [23] employed PD theory to simulate a coupled chemical transport and water flow. In this study, the authors implemented the BB-PD theory to formulate chemical transport and water flow by taking into account diffusion, advection, and dispersion processes in partially saturated porous media.

2.3.1. Chemical Transport in the Realm of PD. Similar to the water flow due to gravity and heat flow due to temperature gradient, diffusion of chemicals moves from high to low potential. Therefore, we can describe Fick's law in PD framework for chemical transport in a similar way to what we did for PD based Fourier's equation of heat diffusion.

$$
\frac{\partial C_{i}}{\partial t}=\dot{C}_{i}=\int_{H_{i}}\left(\underline{R}_{i} \mathbf{r}_{i j}-\underline{R}_{j} \mathbf{r}_{j i}\right) \mathrm{d} V_{j}+\left(\theta_{m}\right)_{i},
$$

where

$$
\begin{aligned}
\underline{R} & =\mathbf{J}_{m}^{T} \mathbb{K} \mathbf{r}_{i j} w_{i j}, \\
\frac{\partial C_{i}}{\partial t} & =\dot{C}_{i}=\int_{H_{i}}\left(\left(\mathbf{J}_{m}\right)_{j}^{T} \mathbb{K}_{j}+\left(\mathbf{J}_{m}\right)_{i}^{T} \mathbb{K}_{i}\right) \mathbf{r}_{i j} w_{i j} \mathrm{~d} V_{j}+\left(\theta_{m}\right)_{i} .
\end{aligned}
$$

The discrete form of (29) may be written as

$$
\begin{aligned}
\dot{C}_{i} & =\sum_{j \in H_{i}} \underbrace{\left(\left(\mathbf{J}_{m}\right)_{j}^{T} \mathbb{K}_{j}+\left(\mathbf{J}_{m}\right)_{i}^{T} \mathbb{K}_{i}\right) \mathbf{r}_{i j} w_{i j} V_{j}}_{-\nabla \cdot\left(J_{m}\right)_{i}}+\left(\theta_{m}\right)_{i}, \\
H_{\text {Con }} & =\sum_{j \in H_{i}}\left(C_{j}-C_{i}\right) \mathbf{r}_{i j} w_{i j} V_{j}, \\
\therefore \nabla C_{i} & \left.\longrightarrow \sum_{j \in H_{i}} \mathbf{r}_{i j} \otimes \mathbf{r}_{i j} w_{i j} V_{j}\right]^{-1}\left[\sum_{j \in H_{i}}\left(C_{j}-C_{i}\right) \mathbf{r}_{i j} w_{i j} V_{j}\right], \\
\nabla \cdot\left(\mathbf{J}_{m}\right)_{i} & \longrightarrow \sum_{j \in H_{i}}\left(\left(\mathbf{J}_{m}\right)_{j}^{T} \mathbb{K}_{j} r_{j i}-\left(\mathbf{J}_{m}\right)_{i}^{T} \mathbb{K}_{i} \mathbf{r}_{i j}\right) w_{i j} V_{j}, \\
\frac{\partial C_{i}}{\partial t} & =-\nabla \cdot \mathbf{J}_{m}+\theta_{m},
\end{aligned}
$$

where $\mathbf{J}_{m}=-D \nabla C$ is the flux of solute, $D$ is the diffusivity of solute in solvent, $C$ is the concentration of solute, and $\theta_{m}$ is the rate of generation of solute per unit volume of the solvent.

2.3.2. Bond-Based Peridynamic (PD) Chemical Transport. In a bond-based PD model, point $i$ interacts with neighboring material points $j$ in its domain in a pairwise manner. The change in chemical concentration at the two end points of a bond is assumed to cause the chemical to flow along the axis of the bond only. The pairwise interaction of material points is written as follows:

$$
\begin{gathered}
\frac{\partial C_{i}}{\partial t}=\dot{C}_{i}=\int_{H_{i}} f_{C} \mathrm{~d} V_{j}+\left(\theta_{m}\right)_{i}, \\
\dot{C}_{i}=\int_{H_{i}}\left(d \frac{C_{j}-C_{i}}{\mathbf{r}_{i j}}\right) \mathrm{d} V_{j} .
\end{gathered}
$$

The response function of diffusion is designated by $f_{C}$ and expressed as

$$
f_{C}\left(\mathbf{r}_{i j}, t\right)=d \frac{C_{j}-C_{i}}{\mathbf{r}_{i j}}
$$

where $d=\widehat{D} / V_{H_{i}}$ is micro diffusivity of the associated chemical bonds that connect points $i$ and $j$ as shown in Figure 2, $V_{H_{i}}$ is the horizon volume of material point centered at $i$, and $\widehat{D}$ is the PD diffusivity of chemical bonds between material points $i$ and $j$.

In order to create a linkage between the PD properties and the classical material properties, we directly borrow expressions from [8] and coined them as chemical transport phenomena. Thus, for one-, two-, and three-dimensional analysis, the PD micro diffusivities are expressed, respectively, as follows: $d$ is defined in terms of the classical diffusivity $D$ as

$$
\begin{cases}d=\frac{2 D}{A \delta^{2}} ; & \text { for }(1-D), \\ d=\frac{6 D}{\pi h \delta^{3}} ; & \text { for }(2-D), \\ d=\frac{6 D}{\pi h \delta^{4}} ; & \text { for }(3-D),\end{cases}
$$

where $A, h, D$, and $\delta$ are cross-sectional area, thickness, diffusivity of solute, and horizon, respectively.

For the examples that follow, the PD heat conduction equation has been solved numerically by replacing the nonlocal integral equation (11) with finite sum:

$$
\left(\rho C_{v}\right)_{i}\left(\dot{T}_{i}\right)^{n}=\sum_{j \in H_{i}}\left(k \frac{\left(T_{j}\right)^{n}-\left(T_{j}\right)^{n}}{\mathbf{r}_{i j}}\right) \mathrm{d} V_{j},
$$

where $n$ signifies the number of time steps, $\mathrm{i}$ denotes the point of interest, and $\mathrm{j}$ is the point in the horizon of $\mathrm{i} . V_{j}$ is the volume subdomain related to the material point $j$. The forward difference computational scheme has been employed by solving the following equation:

$$
T_{(i)}^{n+1}=T_{(i)}^{n}+\frac{\Delta t}{\left(\rho C_{v}\right)_{i}} \sum_{j \in H_{i}}\left(k \frac{\left(T_{j}\right)^{n}-\left(T_{j}\right)^{n}}{\mathbf{r}_{i j}}\right) \mathrm{d} V_{j} .
$$

\section{Case Studies}

In this section, we implemented the state-based PD approach to illustrate the versatility of the PD formulation. Five 


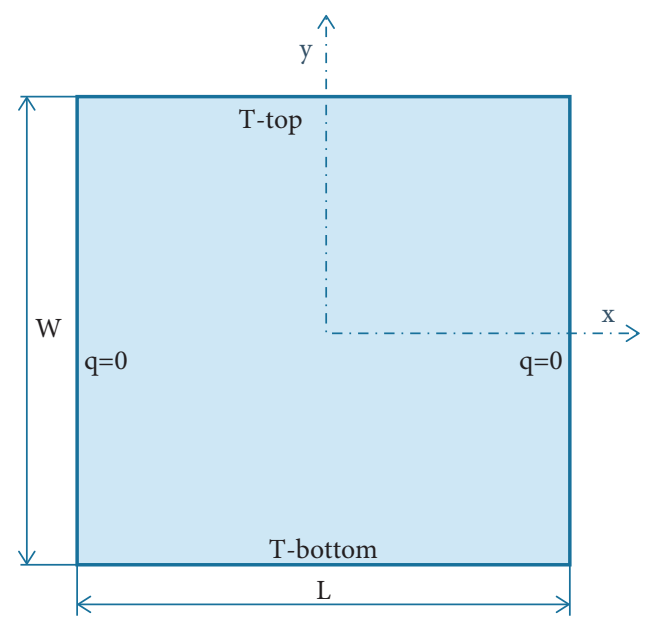

FIgURE 6: Model geometry of square plate.

TABLe 1: Material properties and dimensions.

Geometric parameters Material properties

Length, $L=2 \mathrm{~cm}$

Width, $W=2 \mathrm{~cm}$ Thermal conductivity $\kappa=1.6 \mathrm{~W} / \mathrm{K} . \mathrm{m}$

Thickness, $t=0.01 \mathrm{~cm}$ Heat capacity $C_{v(A)}=154.4 \mathrm{~J} / \mathrm{K} . \mathrm{kg}$ Density $\rho_{(A)}=7740 \mathrm{~kg} / \mathrm{m}^{3}$

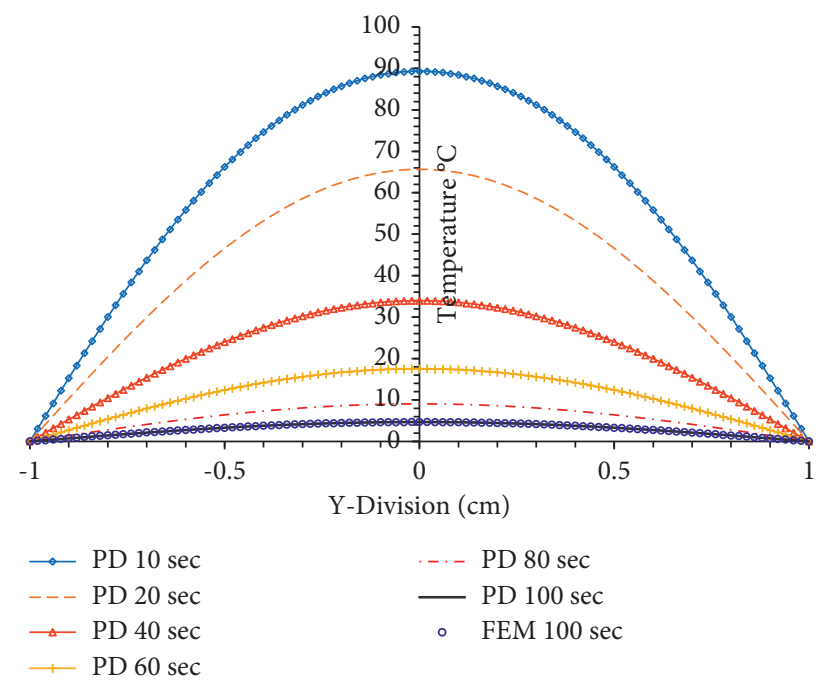

FIgURE 7: Temperature values for symmetric boundary conditions.

illustrative examples are simulated and presented; the first example illustrates two-dimensional heat conduction with symmetric boundary condition. The effect of nonsymmetrical boundary condition is exemplified in the second example. The third and fourth examples demonstrate the beauty of PD theory in dealing with discontinuities. Finally, a single pellet of $\mathrm{Bi}_{2} \mathrm{Te}_{3}$ thermoelectric material has been considered to compute the temperature and voltage values.

Example 1. In this example, square plate with its dimensions that are shown in Figure 6 and material properties that are shown in Table 1 has been considered. The plate is subjected to the following symmetric boundary and initial conditions:
Boundary conditions:

$$
\begin{aligned}
T\left(\frac{W}{2}, y, t\right) & =0^{\circ} \mathrm{C}, \\
T\left(-\frac{W}{2}, y, t\right) & =0^{\circ} \mathrm{C}
\end{aligned}
$$

Initial conditions:

$$
T(x, y, 0)=100^{\circ} \mathrm{C},-\frac{L}{2} \leq x \leq \frac{L}{2},-\frac{W}{2} \leq y \leq \frac{W}{2} .
$$

The domain in this demonstrating example is discretized into 20 by 20 nodal points in the $x$ and the $y$ directions, 

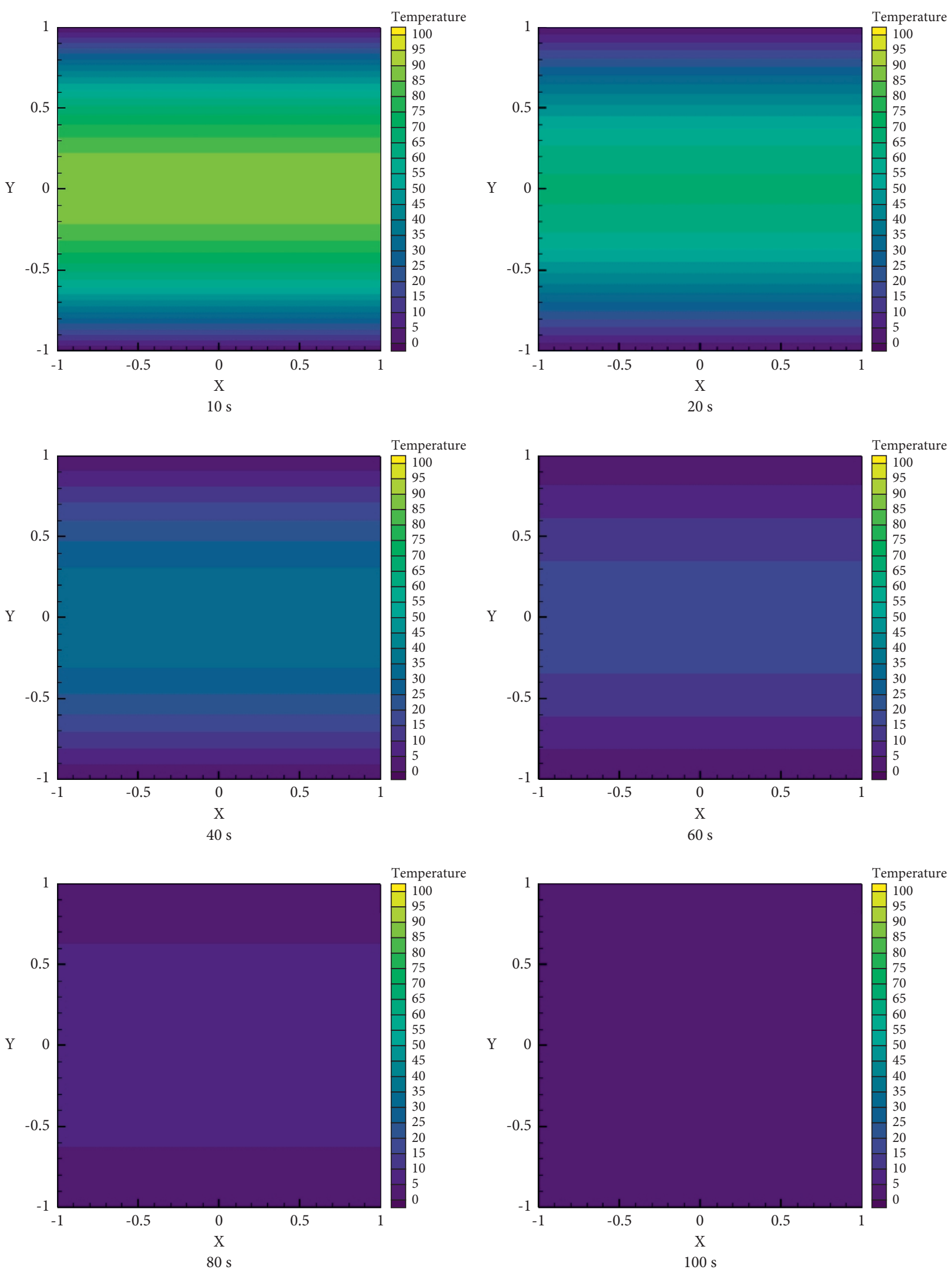

Figure 8: Temperature contours for time values of (a) 10, (b) 20, (c) 40, (d) 60, (e) 80, and (f) 100 seconds. 


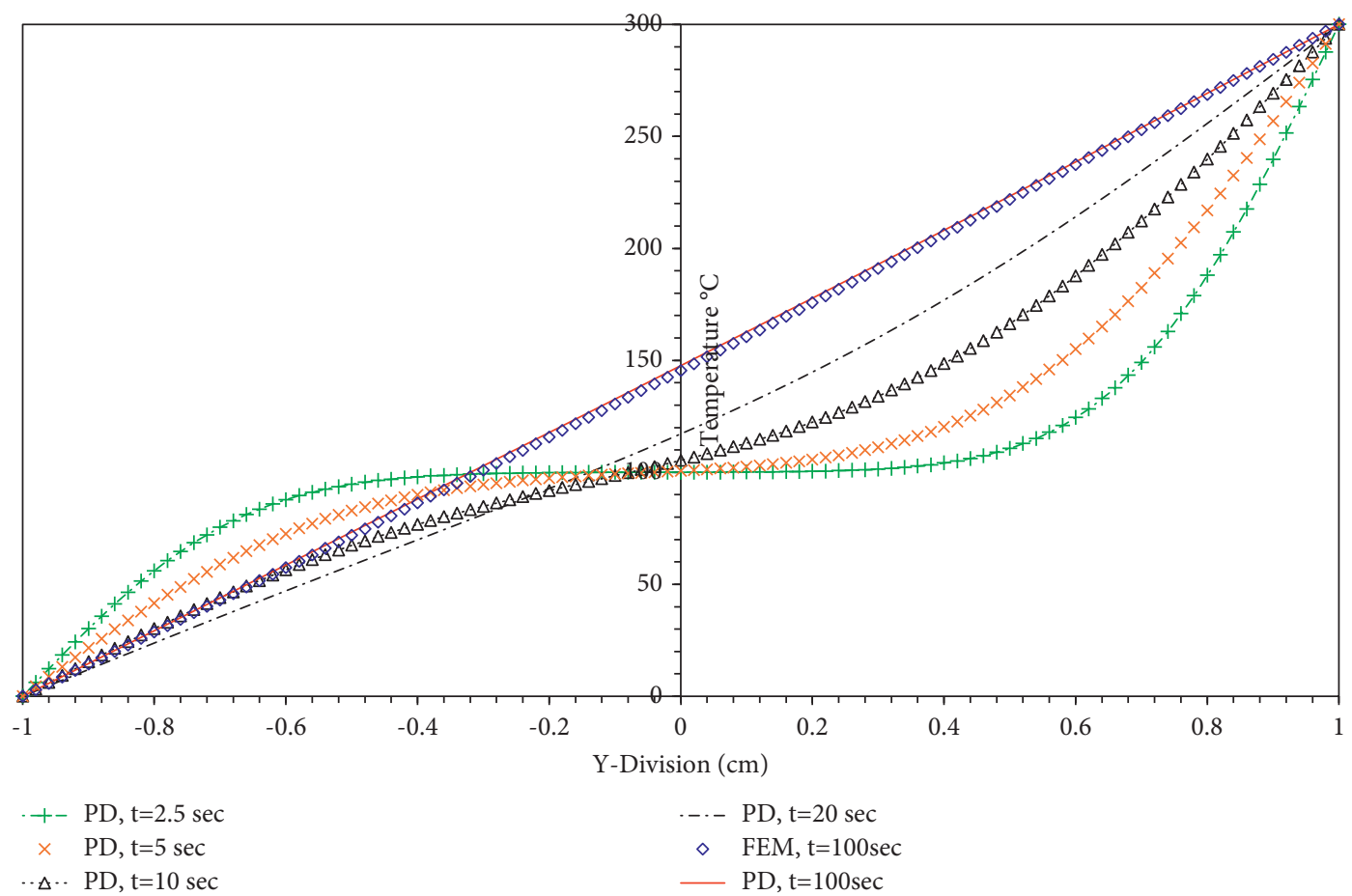

Figure 9: Temperature values for nonsymmetric boundary conditions.

respectively, with time step of $10^{-2}$ seconds. Figure 7 illustrates PD and FEM comparisons of two-dimensional temperature variations. As presented in Figure 7, the temperature decreases with time and reaches its steady-state value. Further, it is found that PD results are in close agreement with those of FEM counterpart.

From Figure 8, we also noticed a decrease in temperature inside the plate as the time goes by. The temperature in the plate was initially $100^{\circ} \mathrm{C}$, and $0^{\circ} \mathrm{C}$ was imposed at the bottom and top boundaries. Figure 8 illustrates the temperature contour plot for time $t=10 \mathrm{~s}, t=20 \mathrm{~s}, 40 \mathrm{~s}, 60 \mathrm{~s}, 80 \mathrm{~s}$, and $100 \mathrm{~s}$.

Example 2. Heat conduction with nonsymmetric boundary.

In this example, a temperature of $0^{\circ} \mathrm{C}$ was enforced at the bottom of the plate and $300^{\circ} \mathrm{C}$ at the top, and the temperature on the rest of the plate was subjected to initial value of $100^{\circ} \mathrm{C}$ as shown below:

Initial conditions:

$$
T(x, y, 0)=100^{\circ} \mathrm{C},-\frac{L}{2} \leq x \leq \frac{L}{2},-\frac{W}{2} \leq y \leq \frac{W}{2}
$$

Boundary conditions:

$$
\begin{aligned}
T\left(\frac{W}{2}, y, t\right) & =300^{\circ} \mathrm{C}(\text { Top }), \\
T\left(-\frac{W}{2}, y, t\right) & =0^{\circ} \mathrm{C}(\text { Bottom }) .
\end{aligned}
$$

The temperature field across the plate is illustrated in Figure 9. The temperature distribution inside the plate becomes closer to linear distribution as simulation time increases. In this example, we also compared temperature values from PD and FEM at $t=100 \mathrm{~s}$. As can be seen from Figure 9, temperature distributions tend to be interestingly closer. Further, Figure 10 depicts the temperature contour of the plate up to 100 -second simulation time. Therefore, from the above two examples, we may draw the conclusion that PD theory is an interesting theory that can deal with diffusion problems correctly.

Example 3. Adiabatic crack with constant heat flux perpendicular to crack surface.

To validate the proposed PD method in handling discontinuities, we considered here an inclined crack with adiabatic inclined crack as shown in Figure 11. In this example, the dimensions are the same as the previous examples and $\beta=45^{\circ}$. Equal and opposite magnitude of temperature has been imposed on the top and bottom edge of the plate in order to keep the heat flux constant. In the meantime, the left and right edges of the plate are heat-insulated. The inclined crack is modeled as an adiabatic crack in order to capture the discontinuity in temperature profile using PD theory as shown in Figure 12.

Figure 12 depicts the comparison between PD results and FEM in the case of adiabatic inclined crack. Figure 12 proves that our PD solution agrees well with the solution from FEM. Hence, our proposed peridynamic model is able 

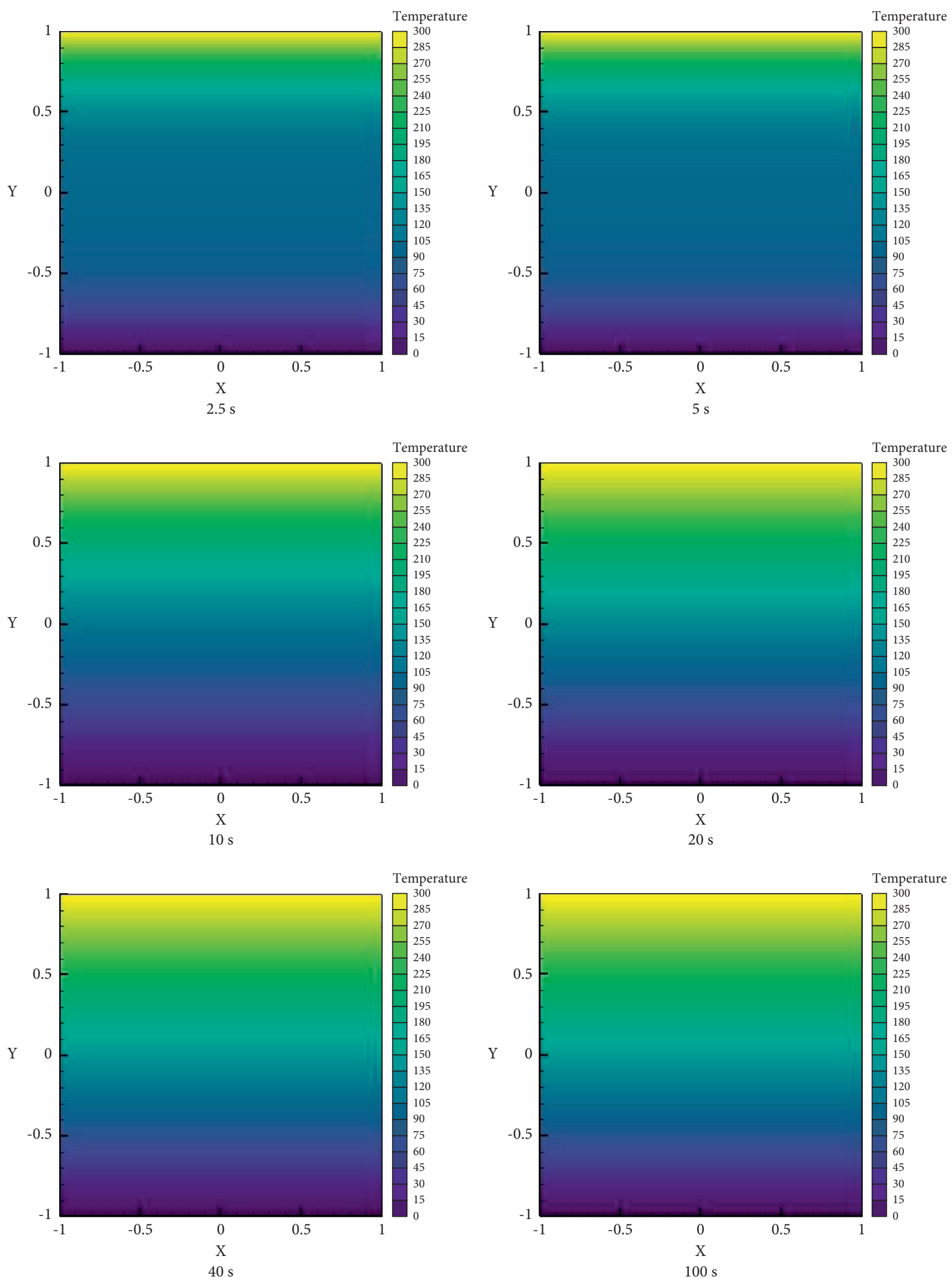

Figure 10: Temperature contours for time values of (a) 2.5, (b) 5. (c) 10, (d) 20, (e) 40, and (f) 100 seconds. 


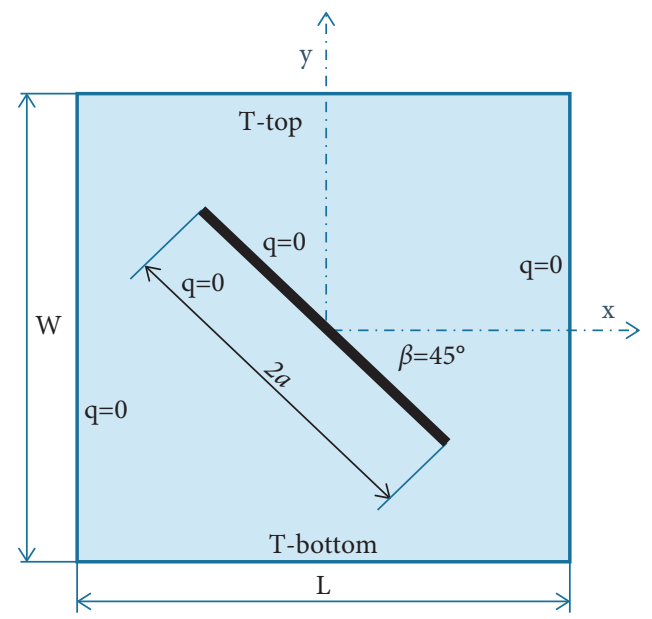

FIgURE 11: Model geometry and boundary conditions for a square plate with adiabatic inclined crack.

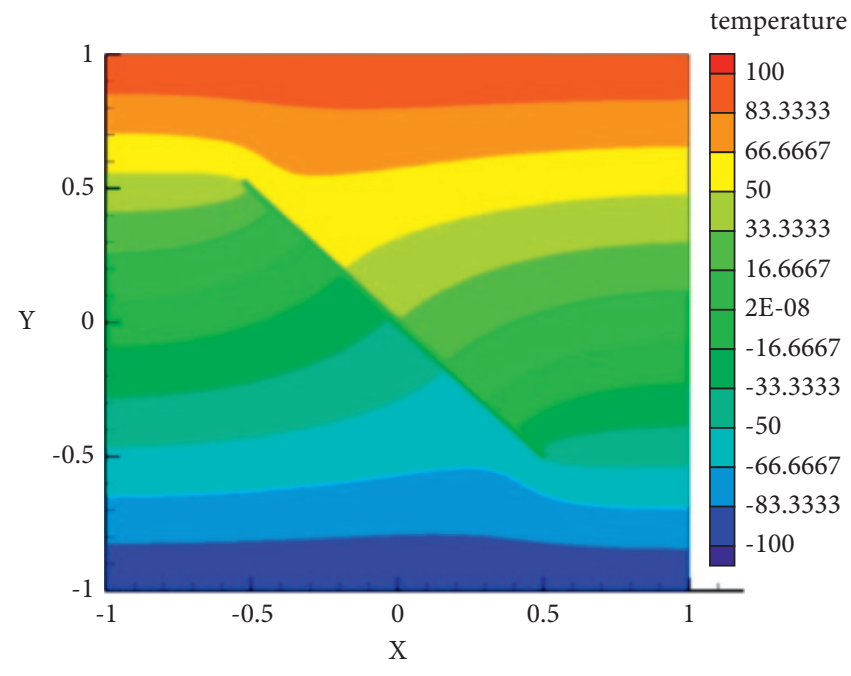

(a)

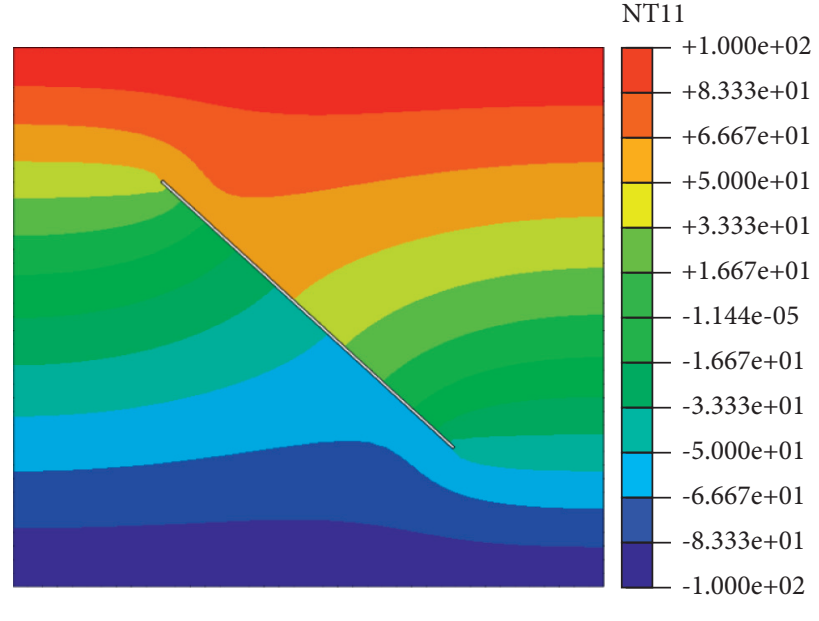

(b)

FIgURE 12: A comparison of temperature contour in case of adiabatic inclined crack when $t=100 \mathrm{~s}$ and at $X=0$ or (L/2). (a) PD. (b) FEM.

to capture the transient heat conduction of a plate with an insulated inclined crack.

Further, Figure 13 illustrates the PD and FEM temperature variations along the $Y$-axis for $t=100 \mathrm{~s}$. As we can see from Figure 13, PD and FEM results are in close agreement.

Example 4. Isothermal crack with specified temperature at the crack surface.

This example considers an inclined crack with isothermal crack condition as shown in Figure 14. Once again, the dimensions are the same as the previous examples and $\beta=45^{\circ}$. Here, the temperature values of equal magnitude are specified at the four edges of the plate, and an essential boundary condition is defined on the crack surface. For this set of boundaries, we choose $T_{2}>T_{1}$.
Similar to Example 3, we compared our PD results with those of FEM. It is also observed from Figures 15 and 16 that our solution is consistent with solution from FEM.

Example 5. Single pellet of bismuth telluride $\left(\mathrm{Bi}_{2} \mathrm{Te}_{3}\right)$.

The main target of this example is to show the effectiveness of PD theory in dealing with couple fields. A comparison between $\mathrm{PD}$ solution and results from literature [90] has been considered by taking into account constant material properties and temperature dependent material properties of bismuth telluride $\left(\mathrm{Bi}_{2} \mathrm{Te}_{3}\right)$.

Case 1. Constant material properties.

Material properties and model geometry are given in Table 2 and Figure 17 [90], respectively. The problem 


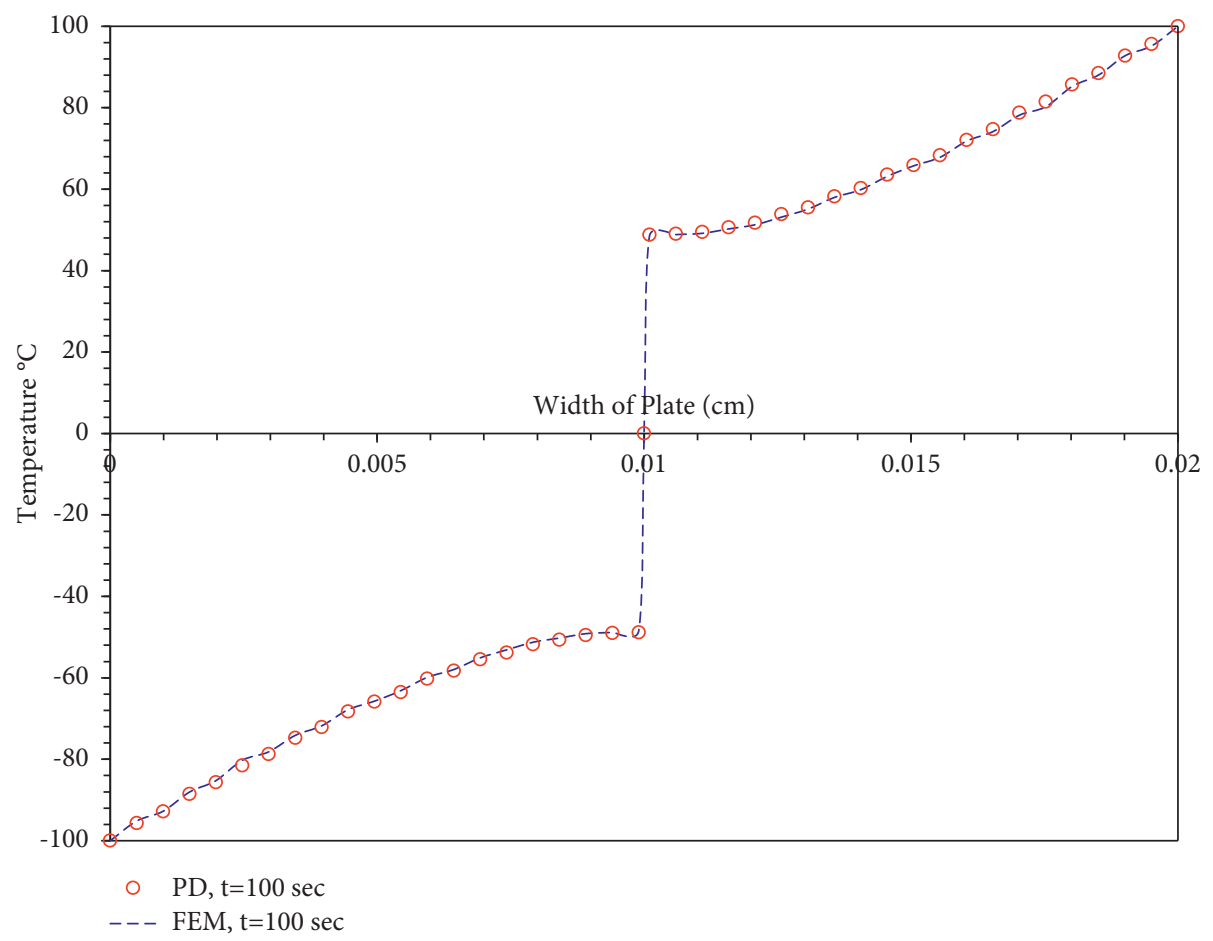

Figure 13: Comparison of PD and FEM solutions at $t=100 \mathrm{~s}$.

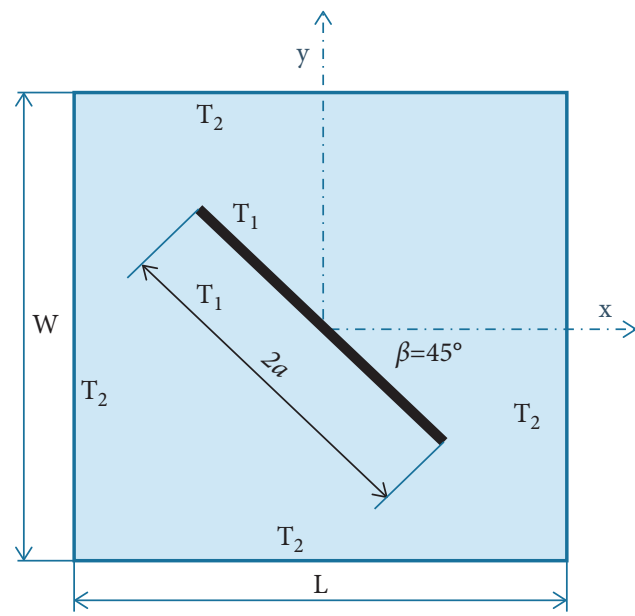

FIgURE 14: Square plate domain with an inclined crack: isothermal crack $T_{2}>T_{1}$.

considered in this example is enforced as one-dimensional linear problem [90].

Temperature and voltage boundary conditions are enforced as

$$
\begin{aligned}
T(0, t) & =273^{\circ} \mathrm{K}, \\
T(L, t) & =298^{\circ} \mathrm{K}, \\
V(L) & =0 \mathrm{v} .
\end{aligned}
$$

Considering constant material properties, temperature results from PD simulation have been obtained and compared with results from [90] as depicted in Figure 18. From Figure 18, it is observed that PD results quite agree with those from [90].

A further study on electric potential values using PD has been conducted. Simulation results from PD and those of from [90] have been compared in Figure 19. It is clear 


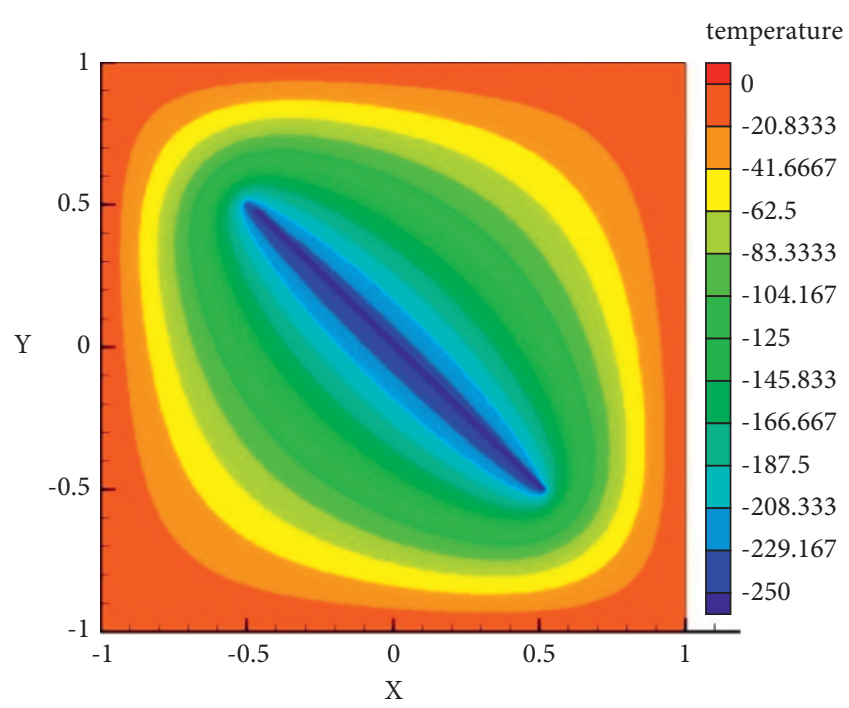

(a)

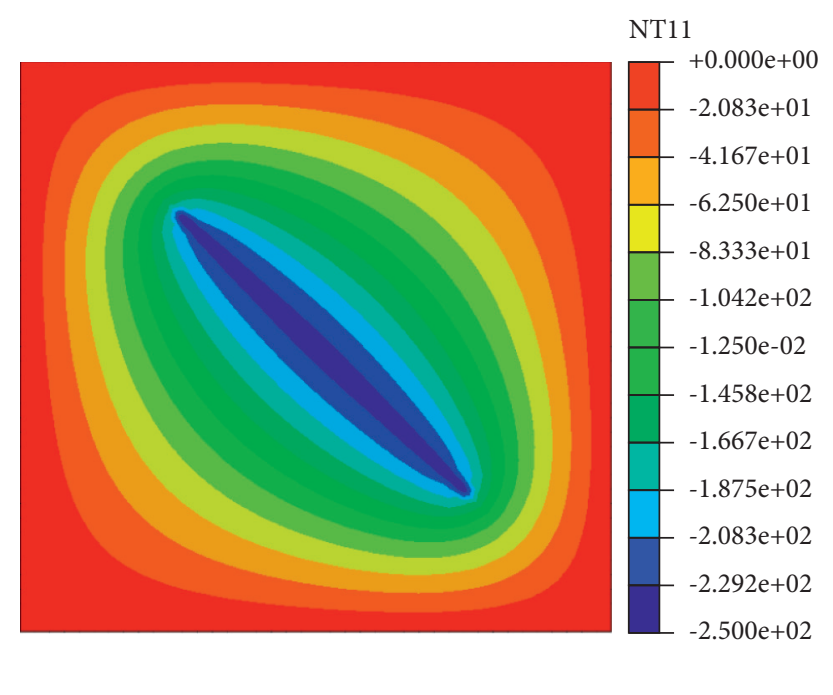

(b)

FIGURE 15: Temperature contour in case of isothermal inclined crack at $t=100 \mathrm{~s}$ : (a) PD solution; (b) FEM solution.

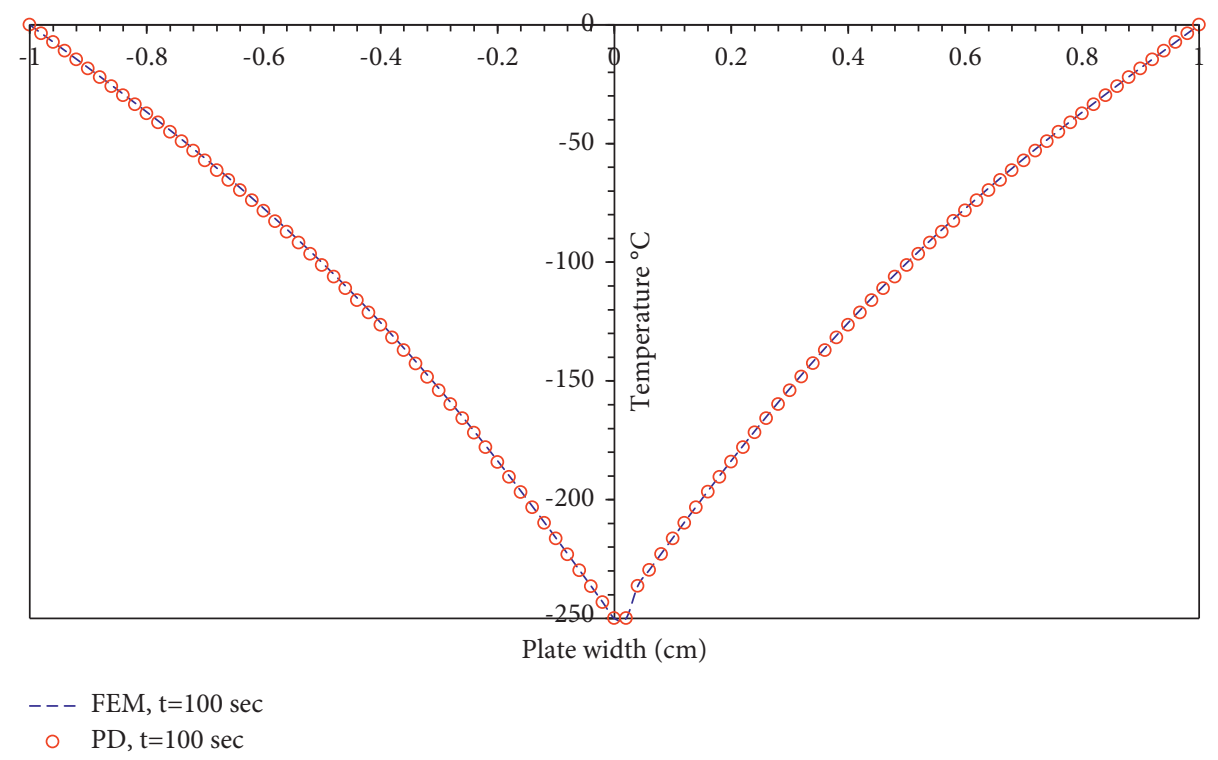

Figure 16: Temperature in case of isothermal inclined crack at $t=100 \mathrm{~s}$, along $X=0$ or $Y=0$.

TABLE 2: Geometric dimensions and material properties [90].

\begin{tabular}{lr}
\hline Geometric parameters & Material properties \\
\hline Length, $L=1.524 \mathrm{~mm}$ & $\alpha=1.849 \times 10^{-4} \mathrm{v} / \mathrm{K}$ \\
Width, $W=1.4 \mathrm{~mm}$ & $\kappa=1.701 \mathrm{~W} / \mathrm{km}$ \\
\hline
\end{tabular}

from Figure 19 that our PD results agreed well with those from [90].

Case 2. Temperature dependence of material properties.

Table 3 depicts the dimensions of model geometry and material properties [90]. Similar to Case 1, temperature and electric potential values have been computed using PD. Boundary conditions and dimensions are similar to Case 1.
Here, we also made a comparison between PD temperature values and those from [90]. As can be seen from Figure 20, PD results smoothly agree with those from [90].

Temperature dependent electric potential values using PD have been computed and compared with results from [90]. It is quite evident from Figure 21 that PD results agree very well with those from [90]. 


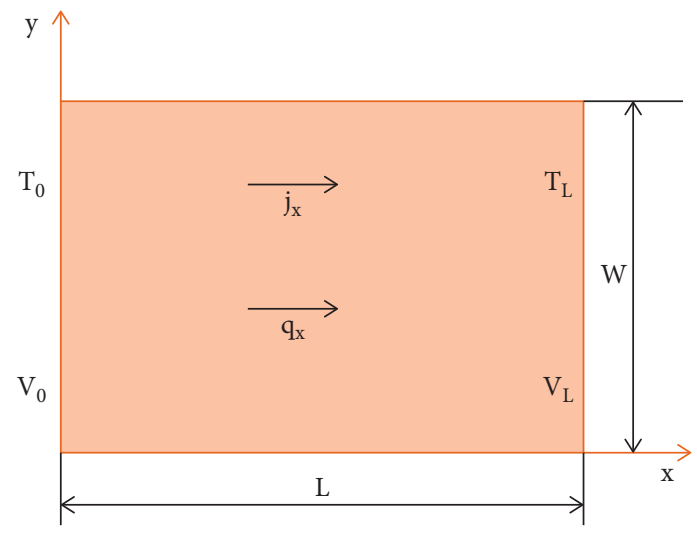

FIGURE 17: Model geometry and boundary conditions [90].

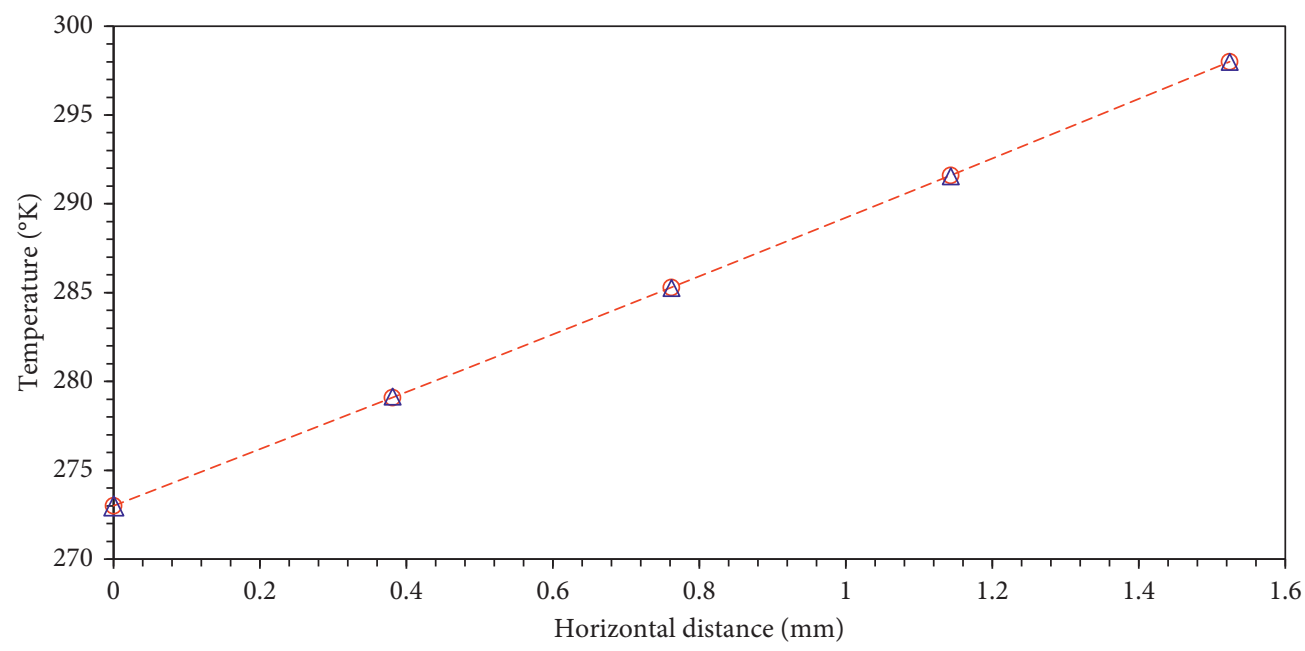

$\triangle \mathrm{PD}$

$-\ominus-$ Ref.

Figure 18: Comparison of temperature values from PD and [90].

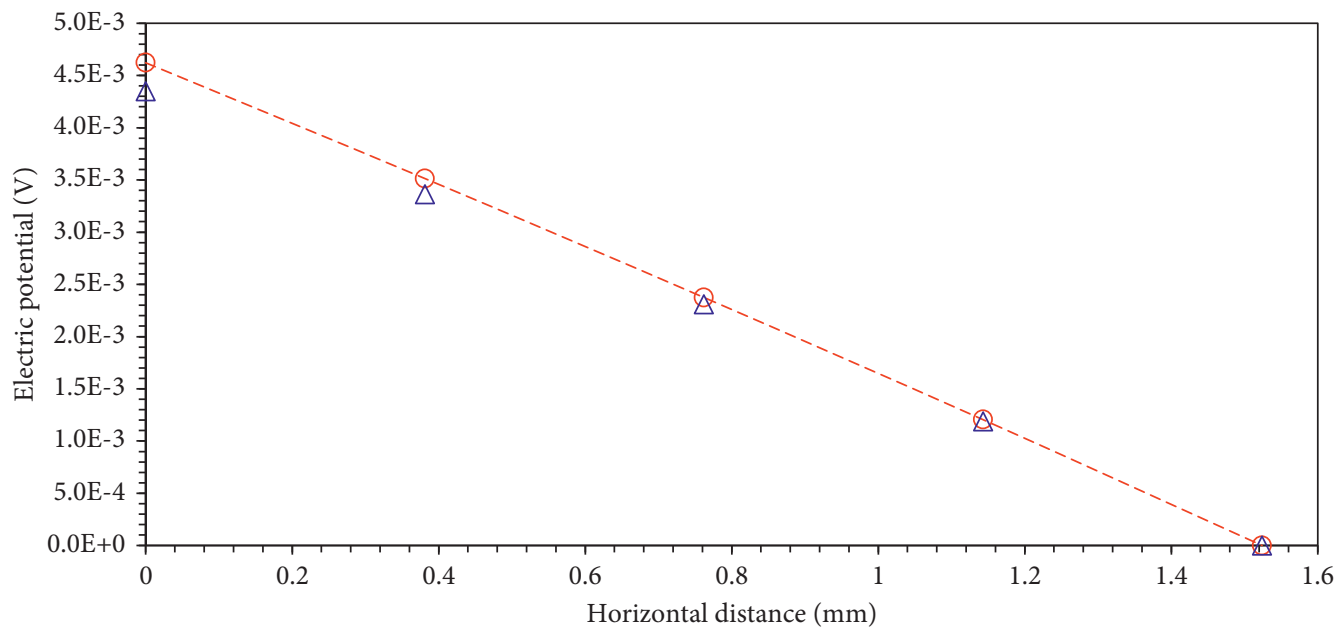
$\triangle \mathrm{PD}$
$-\ominus-$ Ref.

Figure 19: Comparison of potential values from PD and [90]. 
TABLE 3: Dimensions and material properties (temperature dependent).

\begin{tabular}{lc}
\hline Geometric parameters & Material properties \\
\hline Length, $L=1.524 \mathrm{~mm}$ & $\alpha=1.804 \times 10^{-4}+3.598 \times 10^{-7}(T-273)$ \\
Width, $W=1.4 \mathrm{~mm}$ & $\kappa=1.754-4.260 \times 10^{-3}(T-273)$ \\
\hline
\end{tabular}

$T$ is in Kelvin.

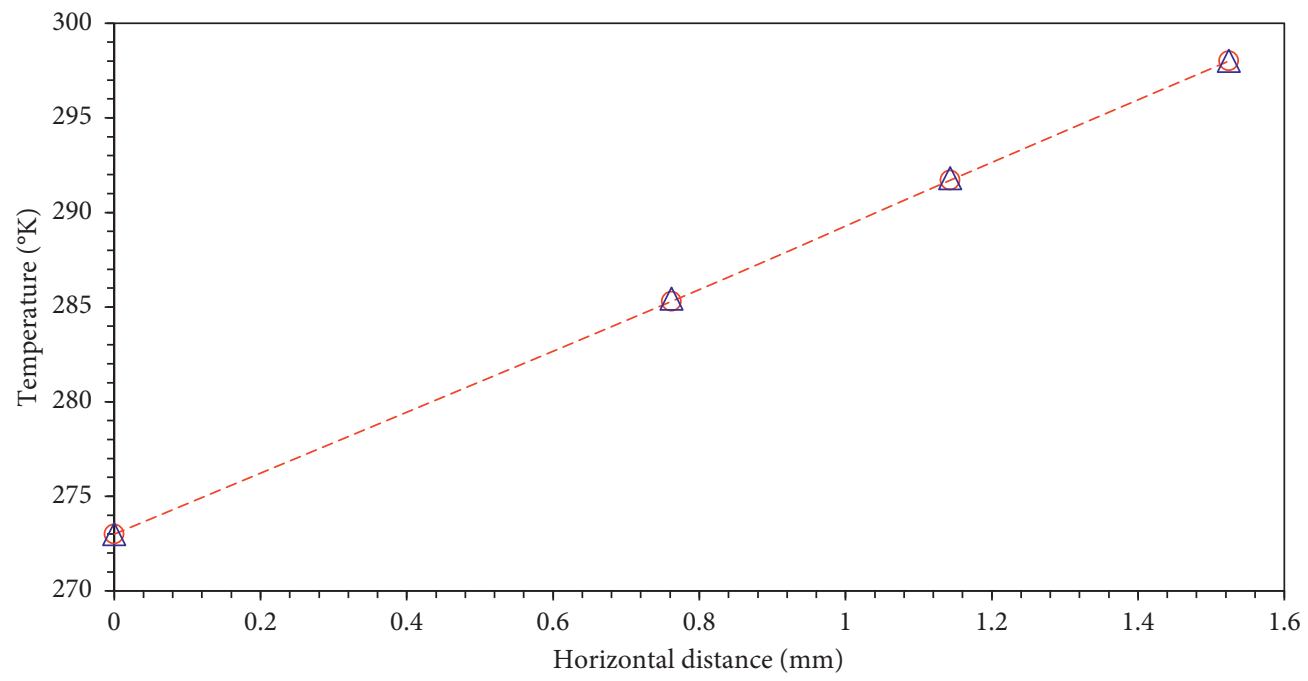

$$
\begin{gathered}
\triangle \text { PD } \\
-\ominus-\text { Ref. }
\end{gathered}
$$

FIGURE 20: Comparison of temperature values in case of temperature dependent material properties from PD and [90].

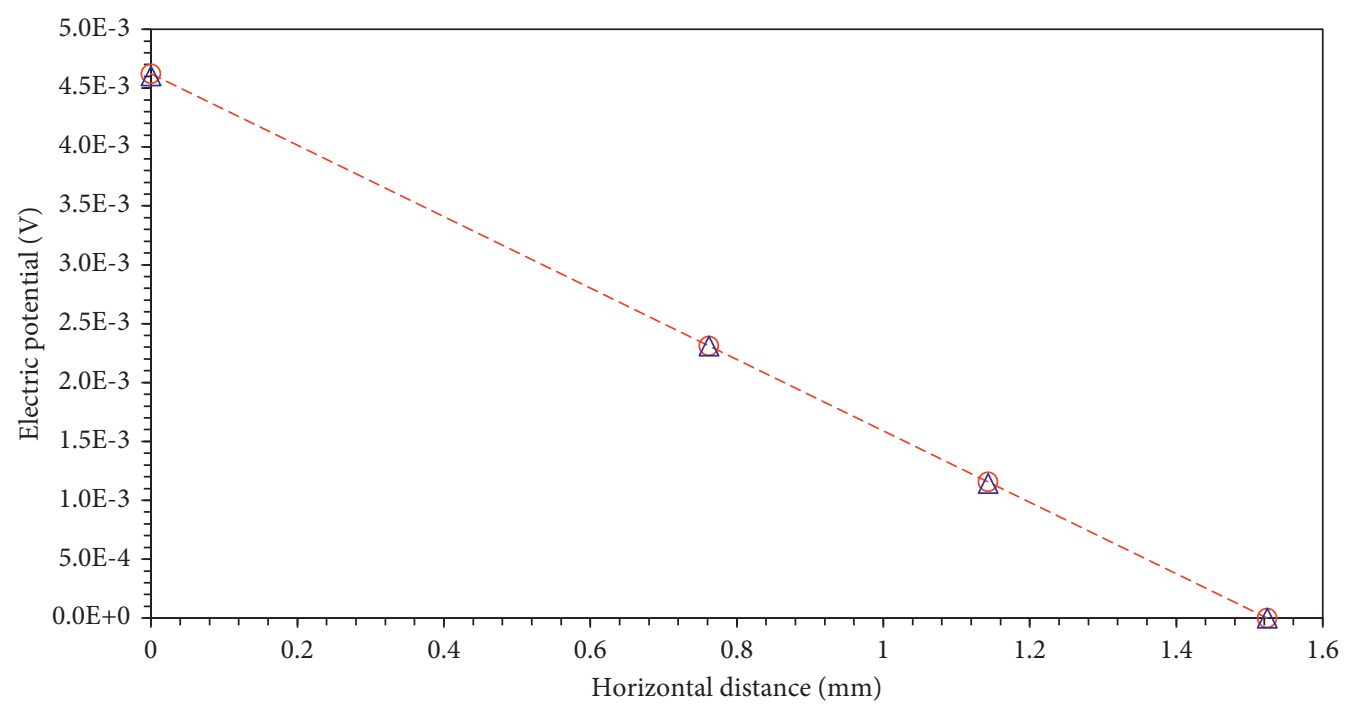

$$
\begin{gathered}
\triangle \text { PD } \\
-\ominus-\text { Ref. }
\end{gathered}
$$

FIGURE 21: Comparison of potential values in case of temperature dependent material properties from PD and [90].

\section{Conclusion}

This article revisited the PD computational scheme that is pertinent to diffusion based problems like heat diffusion, electrical conduction, and chemical transport. In this review, PD functional integrals play a vital role in replacing gradients of hydraulic potentials, solute concentration, temperature, and electrical conduction. This is because functional integrals are valid anywhere in the domain despite the presence of discontinuities. Here, the state-based PD diffusion equation developed by $[7,8]$ has been borrowed to write electrical conduction and chemical transport 
equations. Five examples have been demonstrated to show the versatility of the $\mathrm{PD}$ theory, and the results were compared with results from [90] and FEM. Temperature values inside a square plate have been determined in the first example by considering symmetric temperature boundary. In the second example, nonsymmetric temperature boundary has been considered, and results were presented. In both examples, the PD results have been compared with FEM results, and close agreement has been obtained. In the third and fourth demonstrations, we proved the competence of PD in handling discontinuities. In these examples, results from PD and FEM have been also compared and found to be interesting. Finally, we solved one-dimensional thermoelectric phenomenon by comparing PD results with those from [90] and proved the capability of PD to take care of coupled fields. Hence, we may conclude that PD theory is way beyond solving fracture and solid mechanics problems. It is also versatile in dealing with diffusion based models and their coupled fields.

\section{Conflicts of Interest}

The authors wish to confirm that there are no known conflicts of interest associated with this publication.

\section{References}

[1] M. I. Azis, "Standard-BEM solutions to two types of anisotropic-diffusion convection reaction equations with variable coefficients," Engineering Analysis with Boundary Elements, vol. 105, pp. 87-93, 2019.

[2] S. A. Silling, "Reformulation of elasticity theory for discontinuities and long-range forces," Journal of the Mechanics and Physics of Solids, vol. 48, no. 1, pp. 175-209, 2000.

[3] W. Gerstle and A. Silling, D. Read, V. Tewary, and R. Lehoucq, "Peridynamic simulation of electromigration," Computers, Materials \& Continua, vol. 8, no. 2, pp. 75-92, 2008.

[4] F. Bobaru and M. Duangpanya, "The peridynamic formulation for transient heat conduction," International Journal of Heat and Mass Transfer, vol. 53, no. 19-20, pp. 4047-4059, 2010.

[5] F. Bobaru and M. Duangpanya, "A peridynamic formulation for transient heat conduction in bodies with evolving discontinuities," Journal of Computational Physics, vol. 231, no. 7, pp. 2764-2785, 2012.

[6] X. Tian and Q. Du, "Analysis and comparison of different approximations to nonlocal diffusion and linear peridynamic equations," SIAM Journal on Numerical Analysis, vol. 51, no. 6, pp. 3458-3482, 2013.

[7] A. Agwai, Peridynamic Approach for Coupled Fields, University of Arizona, Tucson, AZ, USA, 2011.

[8] S. Oterkus, E. Madenci, and A. Agwai, "Peridynamic thermal diffusion," Journal of Computational Physics, vol. 265, pp. 71-96, 2014.

[9] Z. Chen and F. Bobaru, "Selecting the kernel in a peridynamic formulation:A study for transient heat diffusion," Computer Physics Communications, 2015, In Press.

[10] A. Katiyar, J. T. Foster, H. Ouchi, and M. M. Sharma, "A peridynamic formulation of pressure driven convective fluid transport in porous media," Journal of Computational Physics, vol. 261, pp. 209-229, 2014.
[11] R. Jabakhanji and R. H. Mohtar, "A peridynamic model of flow in porous media," Advances in Water Resources, vol. 78, pp. 22-35, 2015.

[12] L. J. Wang, J. F. Xu, and J. X. Wang, "The Green's functions for peridynamic non-local diffusion," Proceedings of the Royal Society A: Mathematical, Physical \& Engineering Sciences, vol. 472, no. 2193, Article ID 20160185, 2016.

[13] Y. Liao, L. Liu, Q. Liu, X. Lai, M. Assefa, and J. Liu, "Peridynamic simulation of transient heat conduction problems in functionally gradient materials with cracks," Journal of Thermal Stresses, vol. 40, no. 12, pp. 1484-1501, 2017.

[14] A. Jafari, R. Bahaaddini, and H. Jahanbakhsh, "Numerical analysis of peridynamic and classical models in transient heat transfer, employing Galerkin approach," Heat Transfer-Asian Research, vol. 47, no. 3, pp. 531-555, 2017.

[15] C. Diyaroglu, S. Oterkus, E. Oterkus, and E. Madenci, "Peridynamic modeling of diffusion by using finite-element analysis," IEEE Transactions on Components, Packaging and Manufacturing Technology, vol. 7, no. 11, pp. 1823-1831, 2017.

[16] J. Zhao, Z. Chen, J. Mehrmashhadi, and F. Bobaru, "Construction of a peridynamic model for transient advectiondiffusion problems," International Journal of Heat and Mass Transfer, vol. 126, pp. 1253-1266, 2018.

[17] T. Xue, X. Zhang, and K. K. Tamma, "A two-field state-based peridynamic theory for thermal contact problems," Journal of Computational Physics, vol. 374, pp. 1180-1195, 2018.

[18] X. Gu, Q. Zhang, and E. Madenci, "Refined bond-based peridynamics for thermal diffusion," Engineering Computations, vol. 36, no. 8, pp. 2557-2587, 2019.

[19] Y. Tan, Q. Liu, L. Zhang, L. Liu, and X. Lai, "Peridynamics model with surface correction near insulated cracks for transient heat conduction in functionally graded materials," Materials, vol. 13, no. 6, p. 1340, 2020.

[20] L. Guo, X. Zhang, W. Li, and X. Zhou, "Multi-scale peridynamic formulations for chloride diffusion in concrete," Engineering Analysis with Boundary Elements, vol. 120, pp. 107-117, 2020.

[21] A. Shojaei, A. Hermann, P. Seleson, and C. J. Cyron, "Dirichlet absorbing boundary conditions for classical and peridynamic diffusion-type models," Computational Mechanics, vol. 66, no. 4, pp. 773-793, 2020.

[22] B. Wang, S. Oterkus, and E. Oterkus, "Thermal diffusion analysis by using dual horizon peridynamics," Journal of Thermal Stresses, vol. 44, no. 1, pp. 51-74, 2021.

[23] H. Yan, M. Sedighi, and A. P. Jivkov, "Peridynamics modelling of coupled water flow and chemical transport in unsaturated porous media," Journal of Hydrology, vol. 591, Article ID 125648, 2020.

[24] S. A. Silling, M. Zimmermann, and R. Abeyaratne, "Deformation of a peridynamic bar," Journal of Elasticity, vol. 73, no. 1-3, pp. 173-190, 2003.

[25] I. A. Kunin, Elastic Media with Microstructure I: One Dimensional Models, Springer-Verlag, Berlin, Germany, 1982.

[26] T. L. Warren, "A non-ordinary state-based peridynamic method to model solid material deformation and fracture," International Journal of Solids and Structures, vol. 46, pp. 1186-1195, 2009.

[27] S. A. Silling, M. Epton, O. Weckner, J. Xu, and E. Askari, "Peridynamic states and constitutive modelling," Journal of Elasticity, vol. 88, no. 2, pp. 151-184, 2007.

[28] J. O'Grady and J. Foster, "Peridynamic beams: a non-ordinary, state-based model," International Journal of Solids and Structures, vol. 51, no. 18, pp. 3177-3183, 2014. 
[29] C. Xin, "A non-ordinary state based peridynamic modeling of fractures in quasi-brittle materials," International Journal of Impact Engineering, vol. 111, pp. 130-146, 2018.

[30] Y. Ha and F. Bobaru, "Characteristics of dynamic brittle fracture captured with peridynamics," Engineering Fracture Mechanics, vol. 78, pp. 1156-1168, 2011.

[31] F. Li, J. Pan, and C. Sinka, "Modelling brittle impact failure of disc particles using material point method," International Journal of Impact Engineering, vol. 38, pp. 653-660, 2011.

[32] W. Liu and J. W. Hong, "Discretized peridynamics for brittle and ductile solids," International Journal for Numerical Methods in Engineering, vol. 89, pp. 1028-1046, 2012.

[33] E. Postek, T. Sadowski, and M. Boniecki, "Impact of brittle composites: peridynamics modelling," Materials Today: Proceedings, vol. 45, pp. 4268-4274, 2021.

[34] G. Zhang and F. Bobaru, "Modeling the evolution of fatigue failure with peridynamics," The Romanian Journal of Technical Sciences and Applied Mechanics, vol. 61, no. 1, pp. 22-40, 2016.

[35] G. Zhang, Q. Le, A. Loghin, A. Subramaniyan, and F. Bobaru, "Validation of a peridynamic model for fatigue cracking," Engineering Fracture Mechanics, vol. 162, pp. 76-94, 2016.

[36] Y. L. Hu and E. Madenci, "Peridynamics for fatigue life and residual strength prediction of composite laminates," Composite Structures, vol. 160, pp. 169-184, 2017.

[37] J. Jung and J. Seok, "Mixed-mode fatigue crack growth analysis using peridynamic approach," International Journal of Fatigue, vol. 103, pp. 591-603, 2017.

[38] F. Wang, Y. E. Ma, Y. Guo, and W. Huang, "Studies on quasistatic and fatigue crack propagation behaviours in friction stir welded joints using peridynamic theory," Advances in Materials Science and Engineering, vol. 2019, Article ID 5105612, 16 pages, 2019.

[39] S. Bazazzadeh, M. Zaccariotto, and U. Galvanetto, "Fatigue degradation strategies to simulate crack propagation using peridynamic based computational methods," Latin American Journal of Solids and Structures, vol. 16, no. 2, 2019.

[40] L. I. U. Binchao, B. A. O. Rui, and S. U. I. Fucheng, "A fatigue damage-cumulative model in peridynamics," Chinese Journal of Aeronautics, vol. 34, no. 2, pp. 329-342, 2021.

[41] E. Oterkus, I. Guven, and E. Madenci, "Fatigue failure model with peridynamic theory," in Proceedings of the 2010 12th IEEE Intersociety Conference on Thermal and Thermomechanical Phenomena in Electronic Systems, pp. 1-6, Las Vegas, NV, USA, June 2010.

[42] S. A. Silling and A. Askari, "Peridynamic model for fatigue cracking," University of Nebraska, Lincoln, Nebraska, 2014.

[43] E. Madenci, "Combined peridynamics and kinetic theory of fracture for fatigue failure of composites under constant and variable amplitude loading," Theoretical and Applied Fracture Mechanics, vol. 112, 2021.

[44] T. Nguyen, S. Oterkus, and E. Oterkus, "An energy-based peridynamic model for fatigue cracking," Engineering Fracture Mechanics, vol. 241, Article ID 107373, 2021.

[45] J. Han and W. Chen, "An ordinary state-based peridynamic model for fatigue cracking of ferrite and pearlite wheel material," Applied Sciences, vol. 10, no. 12, p. 4325, 2020.

[46] N. Zhu, C. Kochan, E. Oterkus, and S. Oterkus, "Fatigue analysis of polycrystalline materials using Peridynamic Theory with a novel crack tip detection algorithm," Ocean Engineering, vol. 222, Article ID 108572, 2021.

[47] X. Ma, "A 2D peridynamic model for fatigue crack initiation of railheads," International Journal of Fatigue, vol. 135, Article ID 105536, 2020.
[48] Y. Liu, L. Deng, W. Zhong, J. Xu, and W. Xiong, "A new fatigue reliability analysis method for steel bridges based on peridynamic theory," Engineering Fracture Mechanics, vol. 236, Article ID 107214, 2020.

[49] F. Baber and I. Guven, "Solder joint fatigue life prediction using peridynamic approach," Microelectronics Reliability, vol. 79, pp. 20-31, 2017.

[50] Y. Yu and H. Wang, "Peridynamic analytical method for progressive damage in notched composite laminates," Composite Structures, vol. 108, pp. 801-810, 2014.

[51] Y. Zhang and P. Qiao, "A fully-discrete peridynamic modeling approach for tensile fracture of fiber-reinforced cementitious composites," Engineering Fracture Mechanics, vol. 242, Article ID 107454, 2021.

[52] A. Jenabidehkordi, R. Abadi, and T. Rabczuk, "Computational modeling of meso-scale fracture in polymer matrix composites employing peridynamics," Composite Structures, vol. 253, Article ID 112740, 2020.

[53] B. M. Baykan, U. Yolum, E. Özaslan, M. A. Güler, and B. Yıldırım, "Failure prediction of composite open hole tensile test specimens using bond based peridynamic theory," Procedia Structural Integrity, vol. 28, pp. 2055-2064, 2020.

[54] Y. L. Hu, Y. Yu, and E. Madenci, "Peridynamic modeling of composite laminates with material coupling and transverse shear deformation," Composite Structures, vol. 253, Article ID 112760, 2020.

[55] C. Mitts, S. Naboulsi, C. Przybyla, and E. Madenci, "Axisymmetric peridynamic analysis of crack deflection in a single strand ceramic matrix composite," Engineering Fracture Mechanics, vol. 235, Article ID 107074, 2020.

[56] E. Gok, U. Yolum, and M. A. Güler, "Mode II and mixed mode delamination growth in composite materials using peridynamic theory," Procedia Structural Integrity, vol. 28, pp. 2043-2054, 2020.

[57] W. Zhou, D. Liu, and N. Liu, "Analyzing dynamic fracture process in fiber-reinforced composite materials with a peridynamic model," Engineering Fracture Mechanics, vol. 178, pp. 60-76, 2017.

[58] W. Hu, Y. D Ha, and F. Bobaru, "Modeling dynamic fracture and damage in fiber-reinforced composites with peridynamics," International Journal for Multiscale Computational Engineering, vol. 9, pp. 707-726, 2011.

[59] M. Rädel, C. Willberg, and D. Krause, "Peridynamic analysis of fibre-matrix debond and matrix failure mechanisms in composites under transverse tensile load by an energy-based damage criterion," Composites Part B: Engineering, vol. 158, pp. 18-27, 2019.

[60] Y. L. Hu and E. Madenci, "Bond-based peridynamic modeling of composite laminates with arbitrary fiber orientation and stacking sequence," Composite Structures, vol. 153, pp. 139175, 2016.

[61] C. Sun and Z. Huang, "Peridynamic simulation to impacting damage in composite laminate," Composite Structures, vol. 138, pp. 335-341, 2016.

[62] B. Ren, C. T. Wu, P. Seleson, D. Zeng, and D. Lyu, "A peridynamic failure analysis of fiber-reinforced composite laminates using finite element discontinuous Galerkin approximations," International Journal of Fracture, vol. 214, no. 1, pp. 49-68, 2018.

[63] E. Askari, "Peridynamics for multiscale materials modeling," Journal of Physics, vol. 125, pp. 1-11, 2008.

[64] B. Kilic and E. Madenci, "Prediction of crack paths in a quenched glass plate by using peridynamic theory," International Journal of Fracture, vol. 156, pp. 165-177, 2009. 
[65] E. Oterkus and E. Madenci, "Peridynamic theory for damage initiation and growth in composite laminate," Key Engineering Materials, vol. 488, pp. 355-358, 2012.

[66] S. A. Silling, O. Weckner, E. Askari, and F. Bobaru, "Crack nucleation in a peridynamic solid," International Journal of Fracture, vol. 162, no. 1-2, pp. 219-227, 2010.

[67] Y. Ha and F. Bobaru, "Studies of dynamic crack propagation and crack branching with peridynamics," International Journal of Fracture, vol. 162, no. 1-2, pp. 229-244, 2010.

[68] A. Agwai, I. Guven, and E. Madenci, "Predicting crack propagation with peridynamics: a comparative study," International Journal of Fracture, vol. 171, no. 1, pp. 65-78, 2011.

[69] Z. Cheng, G. Zhang, Y. Wang, and F. Bobaru, "A peridynamic model for dynamic fracture in functionally graded materials," Composite Structures, vol. 133, pp. 529-546, 2015.

[70] J. T. Foster, S. A. Silling, and W. W. Chen, "Viscoplasticity using peridynamics," International Journal for Numerical Methods in Engineering, vol. 81, pp. 1242-1258, 2010.

[71] A. Lakshmanan, J. Luo, I. Javaheri, and V. Sundararaghavan, "Three-dimensional crystal plasticity simulations using peridynamics theory and experimental comparison," International Journal of Plasticity, vol. 142, Article ID 102991, 2021.

[72] A. Pathrikar, M. M. Rahaman, and D. Roy, "A thermodynamically consistent peridynamics model for visco-plasticity and damage," Computer Methods in Applied Mechanics and Engineering, vol. 348, pp. 29-63, 2019.

[73] Y. D. Ha, "Dynamic fracture analysis of high-speed impact on granite with peridynamic plasticity," Journal of the Computational Structural Engineering Institute of Korea, vol. 32, no. 1, pp. 37-44, 2019.

[74] O. Weckner and N. A. N. Mohamed, "Viscoelastic material models in peridynamics," Applied Mathematics and Computation, vol. 219, no. 11, pp. 6039-6043, 2013.

[75] R. Delorme, I. Tabiai, L. L. Lebel, and M. Lévesque, "Generalization of the ordinary state-based peridynamic model for isotropic linear viscoelasticity," Mechanics of Time-dependent Materials, vol. 21, no. 4, pp. 549-575, 2017.

[76] E. Madenci and S. Oterkus, "Ordinary state-based peridynamics for thermoviscoelastic deformation," Engineering Fracture Mechanics, vol. 175, pp. 31-45, 2017.

[77] L. Wu, D. Huang, and F. Bobaru, "A reformulated rate-dependent visco-elastic model for dynamic deformation and fracture of PMMA with peridynamics," International Journal of Impact Engineering, vol. 149, Article ID 103791, 2021.

[78] D. Behera, P. Roy, and E. Madenci, "Peridynamic modeling of bonded-lap joints with viscoelastic adhesives in the presence of finite deformation," Computer Methods in Applied Mechanics and Engineering, vol. 374, Article ID 113584, 2021.

[79] J. A. Mitchell, "A nonlocal, ordinary, state-based plasticity model for peridynamics," Sandia National Laboratories, Albuquerque, NM, USA, pp. SAND2011-SAND3166, 2011.

[80] J. A. Mitchell, A Non-local, Ordinary-State-Based Viscoelasticity Model for Peridynamics, pp. SAND2011-SAND8064, Sandia National Laboratories, Albuquerque, NM, USA, 2011.

[81] S. Sun and V. Sundararaghavan, "A peridynamic implementation of crystal plasticity," International Journal of Solids and Structures, vol. 51, pp. 3350-3360, 2014.

[82] A. Raymond, S. Wildman, and A. G. George, "A Dynamic electro-thermo-mechanical model of dielectric breakdown in solids using PERIDYNAMICS," Journal of Mechanics of Materials and Structures, vol. 10, no. 5, pp. 613-630, 2015.

[83] N. Prakash and G. D. Seidel, "Electromechanical peridynamics modeling of piezoresistive response of carbon nanotube nanocomposites," Computational Materials Science, vol. 113, pp. 154-170, 2016.

[84] N. Prakash and G. D. Seidel, "Computational electromechanical peridynamics modeling of strain and damage sensing in nanocomposite bonded explosive materials (NCBX)," Engineering Fracture Mechanics, vol. 177, pp. 180-202, 2017.

[85] N. Prakash and G. D. Seidel, "Effects of microscale damage evolution on piezoresistive sensing in nanocomposite bonded explosives under dynamic loading via electromechanical peridynamics," Modelling and Simulation in Materials Science and Engineering, vol. 26, no. 1, Article ID 015003, 2017.

[86] A. Migbar, L. Xin, L. Lisheng, and L. Yang, "Peridynamic formulation for coupled thermoelectric phenomena," Advances in Materials Science and Engineering, vol. 2017, Article ID 9836741, 10 pages, 2017.

[87] A. Migbar, L. Xin, and L. Liu, "Bond based peridynamic formulation for thermoelectric materials," Materials Science Forum, vol. 883, pp. 51-59, 2016.

[88] M. A. Zeleke, X. Lai, and L. Liu, "A peridynamic computational scheme for thermoelectric fields," Materials, vol. 13, no. 11, p. 2546, 2020.

[89] V. Diana and V. Carvell, "An electromechanical micropolar peridynamic model," Computer Methods in Applied Mechanics and Engineering, vol. 365, Article ID 112998, 2020.

[90] B. L. Wang, "A finite element computational scheme for transient and nonlinear coupling thermoelectric fields and the associated thermal stresses in thermoelectric materials," Applied Thermal Engineering, vol. 110, no. 5, pp. 136-143, 2017. 